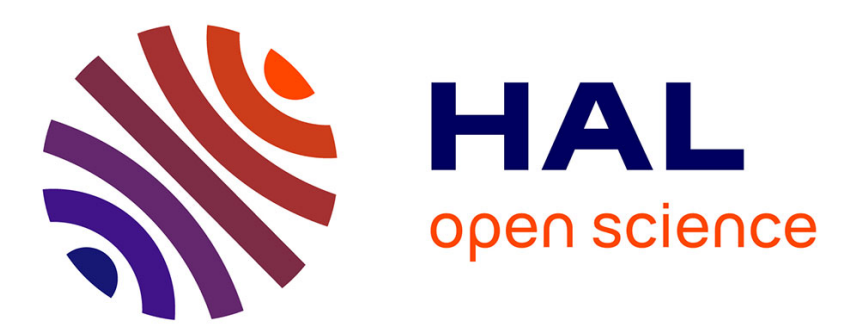

\title{
Arterial stiffness, the hidden face of cardiovascular risk in autoimmune and chronic inflammatory rheumatic diseases
}

\author{
Marie Berger, Pierre Fesler, Camille Roubille
}

\section{- To cite this version:}

Marie Berger, Pierre Fesler, Camille Roubille. Arterial stiffness, the hidden face of cardiovascular risk in autoimmune and chronic inflammatory rheumatic diseases. Autoimmunity Reviews, 2021, 20 (9), pp.102891. 10.1016/j.autrev.2021.102891 . hal-03280802

\section{HAL Id: hal-03280802 https://hal.science/hal-03280802}

Submitted on 29 Jul 2021

HAL is a multi-disciplinary open access archive for the deposit and dissemination of scientific research documents, whether they are published or not. The documents may come from teaching and research institutions in France or abroad, or from public or private research centers.
L'archive ouverte pluridisciplinaire HAL, est destinée au dépôt et à la diffusion de documents scientifiques de niveau recherche, publiés ou non, émanant des établissements d'enseignement et de recherche français ou étrangers, des laboratoires publics ou privés. 


\title{
Arterial stiffness, the hidden face of cardiovascular risk in autoimmune and chronic inflammatory rheumatic diseases
}

\author{
Marie Berger ${ }^{\mathrm{a}}$, Pierre Fesler ${ }^{\mathrm{a}, \mathrm{b}}$, Camille Roubille ${ }^{\mathrm{a}, \mathrm{b}, *}$ \\ ${ }^{a}$ Department of Internal Medicine, CHU Montpellier, Montpellier University, Montpellier, France \\ ${ }^{\mathrm{b}}$ PhyMedExp, University of Montpellier, INSERM U1046, CNRS UMR 9214, Montpellier Cedex 5, France
}

\section{A R T I C L E I N F O}

\section{Keywords:}

Arterial stiffness

Pulse wave velocity

Augmentation index

Chronic inflammatory rheumatic diseases

Rheumatoid arthritis

Systemic lupus erythematosus

Psoriasis

Sjogren's syndrome and ankylosing spondylitis

\begin{abstract}
A B S T R A C T
Background and objective: Cardiovascular diseases (CVD) are the leading causes of death in chronic inflammatory rheumatic diseases and are not solely explained by the increased prevalence of cardiovascular (CV) risk factors in this population. Arterial stiffness, assessed primarily by pulse wave velocity (PWV) and more indirectly by augmentation index (AIx), is a surrogate marker of CVD that should be considered. The objective of this review was to investigate the relationship between arterial stiffness and chronic inflammatory and/or autoimmune diseases.

Methods: We performed a systemic literature review of articles published in Medline from January 2012 to April 2020 restricted to English languages and to human adults. We selected relevant articles about the relationship between arterial stiffness and rheumatoid arthritis, systemic lupus erythematosus, psoriasis, Sjogren's syndrome and ankylosing spondylitis. For each selected article, data on PWV and AIx were extracted and factors that may have an impact on arterial stiffness were identified.

Results: A total of 214 references were identified through database searching and 82 of them were retained for analysis. Arterial stiffness is increased in chronic inflammatory and autoimmune diseases. Traditional CV risk factors such as hypertension and dyslipidemia accentuate this relationship. Current data are insufficient to determine whether disease activity significantly influences arterial stiffness, whereas disease duration seems rather critical. TNF-alpha inhibitors and cardiorespiratory fitness tend to decrease arterial stiffness. Finally, increased arterial stiffness leads to diastolic dysfunction, which is the main mechanism of heart failure in chronic inflammatory rheumatic diseases.

Conclusion: CV risk assessment in chronic inflammatory and autoimmune diseases should also rely on PWV and AIx.
\end{abstract}

\section{Introduction}

Cardiovascular diseases (CVD) are the leading cause of death in chronic inflammatory and autoimmune rheumatic diseases such as rheumatoid arthritis (RA), systemic lupus erythematosus (SLE), and spondyloarthritis [1]. This excess mortality cannot be explained solely by the increased prevalence of cardiovascular (CV) risk factors [2] and the underlying mechanisms leading to such an increased CV risk are not yet clearly understood.

Increasing arterial stiffness is one of the first steps in the atherosclerotic process [3]. Pulse wave velocity (PWV) and augmentation index (AIx) are currently considered independent predictors of major CV events and all-cause mortality [4]. These surrogate markers of subclinical atherosclerosis provide some important prognostic information in addition to traditional CV risk factors [5].

PWV is widely accepted as an accurate and non-invasive method for assessing arterial stiffness in humans [6]. A higher velocity indicates increased arterial stiffness. Currently, the measurement of PWV between the carotid and femoral arteries, which is defined as the aortic PWV, appears to be the most clinically relevant. To date, some studies have supported the prognostic value of aortic PWV measurement as a surrogate marker for subclinical organ damage, in the general population and in patients at increased CV risk [7]. Applanation tonometry is the most useful tool for assessing PWV.

Pulse wave analysis allows us to establish another indirect arterial stiffness parameter: the AIx. While pulse wave spreads in vessels, it

\footnotetext{
* Corresponding author at: Département de Médecine Interne, Hôpital Lapeyronie, 371 avenue du doyen Gaston Giraud, Montpellier cedex 34295 , France. E-mail address: c-roubille@chu-montpellier.fr (C. Roubille).
} 
encounters several caliber disparities. Part of the wave is transmitted, and another part is reflected and returns to the heart. The summation of the "reflected" waves adds to the pulse wave produced by the heart and is responsible for an increase in pressure, normally in the early diastole. The AIx is the ratio of the increased pressure due to the reflected wave and the pulse pressure (that is, the difference between systolic blood pressure and diastolic blood pressure). The greater the arterial stiffness, the faster the reflected wave returns to the heart in systole and the more AIx increases. Thus, systolic blood pressure, ventricular afterload and myocardial oxygen consumption increase, and cardiac coronary perfusion is impaired $[8,9]$.

In recent years, there has been growing interest in the relationship between arterial stiffness, a marker of CV risk, and rheumatic diseases, mainly RA and SLE. Our objective was to conduct a systematic literature research on arterial stiffness in a wide range of chronic inflammatory and autoimmune diseases.

\section{Methods}

\subsection{Data sources and study selection}

We performed a systemic review of articles published from January 2012 to April 2020, restricted to English languages and to human adults, in Medline (via PubMed). We used the MeSH term "Arthritis, Rheumatoid", "Lupus Erythematosus, Systemic" "Psoriasis", "Arthritis, Psoriatic", "Anti-Neutrophil Cytoplasmic Antibody-Associated Vasculitis", "Sjogren's Syndrome", combined with the Boolean operator "AND" with "Vascular Stiffness" or "Pulse Wave Analysis". This last two terms included the concept of arterial stiffness, aortic stiffness, carotid stiffness, wave reflection and augmentation index which were not registered as MeSH term.

We reviewed the abstracts of all the articles we found in the systematic research for relevance. We selected the original articles focusing on the relationship between arterial stiffness and the inflammatory and/ or autoimmune disease of interest. Irrelevant references and reviews were excluded.

\subsection{Data extraction}

The following data were extracted for each article using a predefined data collection form: the study design, sample size, mean age, gender and treatment received by the patients when available. Next, we focused on comparing patients and controls in terms of PWV and AIx. The main findings of each study were collected and factors that had an impact on this association were highlighted.

\section{Results of search}

A total of 214 references were identified through database searching. After reading the abstract, 82 articles were selected for qualitative analysis (Fig. 1). We obtained respectively 42 results for RA (Table 1), 12 for SLE (Table 2), 14 for psoriasis or psoriatic arthritis (Table 3),

9 for spondyloarthritis (Table 4), and $\mathbf{5}$ for Sjögren's syndrome (Table 5).

\section{Discussion}

\subsection{Rheumatoid arthritis (RA) and arterial stiffness}

\subsubsection{Increased arterial stiffness in $R A$}

Among all the studies investigating the relationship between arterial stiffness and RA compared with controls, only two [10,11] found no increase in PWV or AIx. For instance, Anyfanti et al. reported higher levels of PWV among 104 patients with RA, compared to 52 controls $(8.2 \pm 2.1$ vs $7.4 \pm 1.4 \mathrm{~m} / \mathrm{s}, p=0.016)$ [12]. RA patients also showed significantly.

greater values of AIx compared to healthy controls (32.2 \pm 8.6 vs. $28.4 \pm 8.9 \%, P=0.02$ ) [13]. In addition, there appears to be an increase in arterial stiffness in early RA [14].

\subsubsection{Influence of disease characteristics and CV risk factors on arterial stiffness}

Some authors have sought to determine which characteristics may influence arterial stiffness in RA patients. However, evidence is scarce, due to studies with small samples.

First, it has been reported that the duration of the disease may influence arterial stiffness. In a study of 106 RA patients, without CV risk factors, patients with a duration of RA greater than 10 years had a significant increase in PWV compared to those with a recent diagnosis (duration of disease duration $<2$ years) [15]. Interestingly, the authors

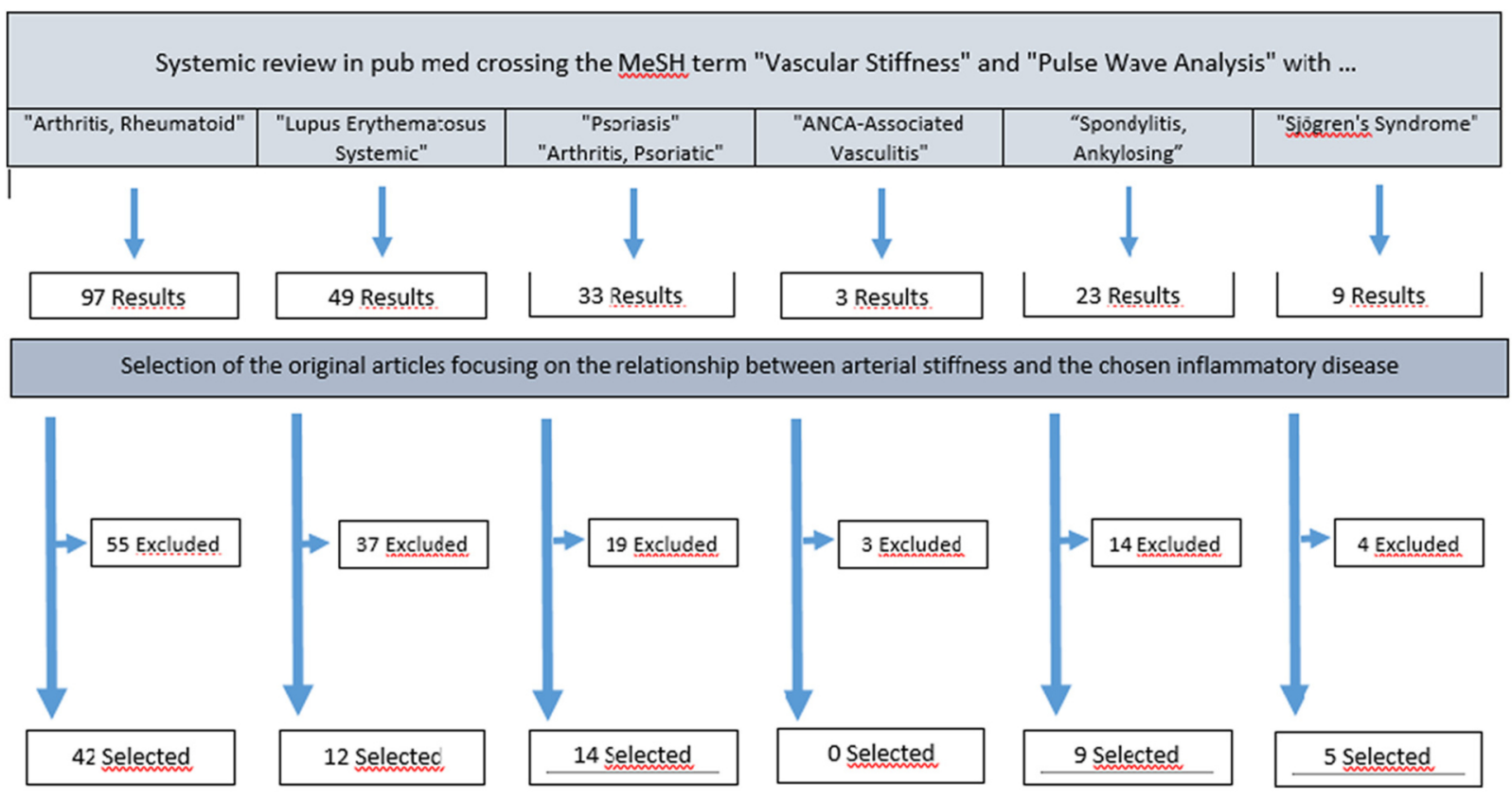

Fig. 1. Flowchart of the selected studies. 
Table 1

Rheumatoid arthritis and arterial stiffness.

\begin{tabular}{|c|c|c|c|}
\hline Reference & Design & Population & Treatment \\
\hline $\begin{array}{l}\text { Anyfanti et al. } 2018 \\
\text { [12] }\end{array}$ & $\begin{array}{l}\text { Cross-sectional } \\
\text { study }\end{array}$ & $\begin{array}{l}\text { Patients } n=104 \\
\text { Age: } 60 \pm 11.4 \text { years Female: } \\
78.8 \% \\
\text { Controls } n=52 \\
\text { Age: } 57.2 \pm 8.2 \text { years Female: } \\
78.8 \%\end{array}$ & $\begin{array}{l}\text { 75\% sDMARD } \\
43 \% \text { bDMARD } \\
27.9 \% \text { CTC }\end{array}$ \\
\hline $\begin{array}{l}\text { Maloberti et al. } 2018 \\
\text { [13] }\end{array}$ & $\begin{array}{l}\text { Cross-sectional } \\
\text { study }\end{array}$ & $\begin{array}{l}\text { Patients } n=55 \\
\text { Age: } 62.8 \pm 8.9 \text { years Female: } \\
73.4 \% \\
\text { Controls } n=55 \\
\text { Age: } 62.8 \pm 8.9 \text { years Female: } \\
73.4 \%\end{array}$ & $\begin{array}{l}52.7 \% \text { CTC } \\
66.6 \% \text { MTX } \\
54.5 \% \text { bDMARD }\end{array}$ \\
\hline $\begin{array}{l}\text { Gunter et al. } 2018 \\
\text { [37] }\end{array}$ & Cross sectional study & $\begin{array}{l}\text { Patients } n=163 \\
\text { Age: } 58.4 \pm 11.7 \text { years Female: } \\
79.1 \%\end{array}$ & $\begin{array}{l}92.6 \% \text { sDMARD or } \\
\text { bDAMRD }\end{array}$ \\
\hline $\begin{array}{l}\text { Şirin Özcan et al. } \\
2017 \text { [16] }\end{array}$ & Cross sectional study & $\begin{array}{l}\text { Patients } n=70 \\
\text { Age: } 52.2 \pm 10.8 \text { years Female: } \\
90 \% \\
\text { Controls } n=35 \\
\text { Age: } 53.5 \pm 6.7 \text { years Female: } 88 \%\end{array}$ & $\begin{array}{l}44 \% \text { SLZ } \\
68 \% \text { HCQ } \\
54 \% \text { CTC } \\
68 \% \text { MTX } \\
8 \% \text { LEF } \\
3 \% \text { bDMARD }\end{array}$ \\
\hline $\begin{array}{l}\text { Vazquez-Del } \\
\text { Mercado et al. } \\
2017 \text { [15] }\end{array}$ & Cohort study & $\begin{array}{l}\text { Patients } n=106 \\
\text { Age: } 46 \pm 12 \text { years Female: } 95.3 \%\end{array}$ & $\mathrm{MD}$ \\
\hline $\begin{array}{l}\text { Aslan et al. } 2017 \\
\text { [19] }\end{array}$ & Cross sectional study & $\begin{array}{l}\text { Patients } n=140 \\
\text { Age: } 52.2 \pm 10.7 \text { years Female: } \\
90 \% \\
\text { Controls } n=140 \\
\text { Age: } 52.7 \pm 8.0 \text { years Female: } 90 \%\end{array}$ & $\begin{array}{l}42.8 \% \text { SLZ } \\
68.5 \% \text { HCQ } \\
54.2 \% \text { CTC } \\
68.6 \% \text { MTX }\end{array}$ \\
\hline $\begin{array}{l}\text { Gunter et al. } 2017 \\
\text { [83] }\end{array}$ & Cohort study & $\begin{array}{l}\text { Patients } n=177 \\
\text { Age: } 58.0 \pm 12.1 \text { years Female: } \\
100 \%\end{array}$ & $\begin{array}{l}90.3 \% \text { sDMARD } \\
11.3 \% \text { bDMARD }\end{array}$ \\
\hline $\begin{array}{l}\text { Robustillo-Villarino } \\
\text { et al. } 2017 \text { [39] }\end{array}$ & Cohort study & $\begin{array}{l}\text { Patients } n=194 \\
\text { Age: } 60.4 \pm 13.2 \text { years } \\
\text { Female: } 75.4 \%\end{array}$ & $\begin{array}{l}\text { 100\% sDMARD } \\
32 \% \text { bDMARD }\end{array}$ \\
\hline $\begin{array}{l}\text { Cypiene et al. } 2017 \\
\text { [22] }\end{array}$ & Cross sectional study & $\begin{array}{l}\text { Patients } n=149 \\
\text { Age: } 48.8 \pm 13.5 \text { years Female: } \\
89.6 \% \\
\text { Controls } n=156 \\
\text { Age: } 42.8 \pm 11.0 \text { years Female: } \\
73.1 \%\end{array}$ & $\begin{array}{l}\text { 48.3\% SLZ } \\
10.3 \% \text { HCQ } \\
17.4 \% \text { CTC } \\
20.1 \% \text { MTX } \\
12.8 \% \text { LEF } \\
26.6 \% \text { bDMARD }\end{array}$ \\
\hline $\begin{array}{l}\text { Scanlon et al. } 2017 \\
\text { [17] }\end{array}$ & Correlative study & $\begin{array}{l}\text { Patients } \mathrm{n}=50 \\
\text { Age: } 57.5 \text { years Female: } 76 \%\end{array}$ & $\begin{array}{l}32 \% \text { CTC } \\
62 \% \text { MTX } \\
42 \% \text { other sDMARD } \\
66 \% \text { bDMARD }\end{array}$ \\
\hline $\begin{array}{l}\text { Cioffi et al. } 2016 \\
\text { [20] }\end{array}$ & Cross sectional study & $\begin{array}{l}\text { Patients } \mathrm{n}=226 \\
\text { Age: } 60 \pm 12 \text { years Female: } 72 \% \\
\text { Controls } n=226 \\
\text { Age: } 60 \pm 12 \text { years Female: } 69 \%\end{array}$ & $\begin{array}{l}35 \% \text { NSAID } \\
11 \% \text { HCQ } \\
45 \% \text { CTC } \\
47 \% \text { MTX } \\
65 \% \text { bDMARD }\end{array}$ \\
\hline $\begin{array}{l}\text { Dzieża- Grudnik } \\
\text { et al. } 2017 \text { [10] }\end{array}$ & Cross sectional study & $\begin{array}{l}\text { Patients } \mathrm{n}=26 \\
\text { Age: } 40 \pm 9.7 \text { years Female: } 80.7 \% \\
\text { Controls } n=29 \\
\text { Age: } 40 \pm 7.6 \text { years Female: } 44.8 \%\end{array}$ & $\begin{array}{l}38 \% \text { NSAID } \\
62 \% \text { CTC }\end{array}$ \\
\hline $\begin{array}{l}\text { Ikdahl et al. } 2016 \\
\text { [84] }\end{array}$ & Longitudinal study & $\begin{array}{l}\text { Patients } n=138 \\
\text { Age: } 59.0[51-66.9] \text { years } \\
\text { Female:76\% }\end{array}$ & $\begin{array}{l}21.7 \% \text { bDMARD } \\
65.2 \% \text { sDMARD } \\
30.4 \% \text { CTC } \\
31.2 \text { NSAID }\end{array}$ \\
\hline Karp et al. 2016 [51] & Cross sectional study & $\begin{array}{l}\text { Patients } \mathrm{n}=31 \\
\text { Age: } 54.7 \pm 14.7 \text { years Female: } \\
100 \% \\
\text { Controls } \mathrm{n}=53 \\
\text { Age: } 53.5 \pm 13.9 \text { years Female: } \\
100 \%\end{array}$ & $\begin{array}{l}6.5 \% \text { AZA } \\
16 \% \text { HCQ } \\
48 \% \text { CTC } \\
71 \% \text { MTX } \\
6 \% \text { LEF } \\
42 \% \text { bDMARD }\end{array}$ \\
\hline $\begin{array}{l}\text { Mitrović et al. } 2017 \\
\text { [23] }\end{array}$ & Cross sectional study & $\begin{array}{l}\text { Patients } n=52 \\
\text { Age: } 60.1 \pm 11.1 \text { years Female: } \\
70.9 \% \\
\text { Controls } n=42\end{array}$ & MD \\
\hline
\end{tabular}

Outcome

PWV in RA $(8.2 \pm 2.1$ vs $7.4 \pm 1.4 \mathrm{~m} / \mathrm{s}, \mathrm{p}=0.016)$

$\nearrow \mathrm{AIx}$ in $\mathrm{RA}(32.2 \pm 8.6$ vs. $28.4 \pm 8.9 \%, p=0.02)$

PWV is not associated with plaque (OR 1.03 [0.66-1.62], $\mathrm{p}=$ $0.9)$.

AIx is not associated with plaque (OR 1.56 CI95\% [0.94-2.70], $p=0.08)$.

PPWV in RA (7.76 vs $6.41 \mathrm{~m} / \mathrm{s}, p=0.009)$.

$\nearrow \mathrm{CMT}$ in $\mathrm{RA}(p=0.0015)$

In multivariate regression, PWV was positively correlated with age $(r=0.450, \mathrm{p}<0.001)$ and RA disease duration $(r=0.340$, $p<0.001)$.

PPW in RA $(7.37 \pm 1.70$ vs $6.49 \pm 0.92 \mathrm{~m} / \mathrm{s}, \mathrm{p}<0.001)$ Seronegative patients had significantly elevated PWV than seropositive $(7.68 \pm 2.24$ vs $7.12 \pm 1.34 \mathrm{~m} / \mathrm{s}, \mathrm{p}=0.031)$.

Factors associated with PP:

- RA duration ( $\mathrm{p}=0.04)$

- RF status ( $\mathrm{p}=0.001$ to 0.003 )

- Leukocytes counts ( $\mathrm{p}=0.02$ to 0.05 )

- Cholesterol ( $p<0.01$ to 0.03 )

TNF- $\alpha$ inhibition was related to reduced PWV

$(\mathrm{p}<0.01)$.

After adjustment, PWV was no longer related to either IMT or plaque.

乃PWV in RA $(8.53 \mathrm{~m} / \mathrm{s} \mathrm{C195 \% [8.28-8.77]} \mathrm{and} 8.01 \mathrm{~m} / \mathrm{s}$ CI95\%[7.77-8.24], $\mathrm{p}=0.008)$.

$\nearrow \mathrm{AIx}$ in RA $(24.81 \% \mathrm{CI} 95 \%$ [23.1-26.45] vs $17.55 \% \mathrm{CI} 95 \%$

[15.97-19.12], $\mathrm{p}<0.0001)$.

AIx correlated with time-averaged tender joint count $(r=0.37$, $\mathrm{p}=0.008)$, CDAI $(\mathrm{r}=0.36, \mathrm{p}=0.01)$, HAQ-II $(r=0.36, \mathrm{p}=$ $0.01)$, swollen joint count $(r=0.36, p=0.10)$.

AoSI was significantly higher in RA patients $(6.3 \pm 4.5$ vs. $4.6 \pm$ $3.5 \%, p<0.001)$.

No differences in PWV between RA and controls (10 CI95\% [8.8-10.9] vs 9.2 CI95\%[8.3-11.4] m/s, p = 0.14). No differences in AIx between RA and controls ( $24.3 \pm 11.5$ vs $10 \pm 12.8 \%, \mathrm{p}=0.22$ ).

Patients with CVD events had significantly higher PWV at baseline compared with those who did not experience CVD events during follow-up $(p<0.001)$.

$\nearrow \mathrm{PWV}$ in RA $(9.2 \pm 4.4$ vs $7.6 \pm 3.0 \mathrm{~m} / \mathrm{s}, p=0.04)$ $\checkmark$ aortic distensibility in RA $(4.4 \pm 4.6$ vs. $5.8 \pm 4.9 \mathrm{kPa}, \mathrm{p}=$ $0.04)$.

$\nearrow$ AoSI in hypentensive RA compared to hypertensive-non RA controls $(0.47 \pm 0.16$ vs $0.38 \pm 0.17, p=0.016)$.

$\nearrow$ cIMT in Hypentensive RA compared to hypertensive-non RA controls $(0.9 \pm 0.26$ vs $0.74 \pm 0.23 \mathrm{~mm}, p<0.001)$. 


\begin{tabular}{|c|c|c|c|c|}
\hline Reference & Design & Population & Treatment & Outcome \\
\hline $\begin{array}{l}\text { Karakulak et al. } 2015 \\
\text { [85] }\end{array}$ & Cross sectional study & $\begin{array}{l}\text { Age: } 56.7 \pm 11.7 \text { years Female: } \\
71.4 \% \\
\text { Patients } n=33 \\
\text { Age: } 54.2 \pm 12.3 \text { years Female: } \\
27.3 \% \\
\text { Controls } n=33 \\
\text { Age: } 52.6 \pm 9.4 \text { years Female: } \\
36.4 \%\end{array}$ & $\begin{array}{l}84.8 \% \text { MTX + LEF } \\
9 \% \text { MTX + HCQ + SLZ } \\
6 \% \text { MTX + ADA }\end{array}$ & $\begin{array}{l}\nearrow \text { AoSI in RA compared to controls }(0.45 \pm 0.12 \text { vs } 0.38 \pm \\
p=0.019) . \\
\text { Nondipper status }(\beta=0.316, P=0.007) \text { was an independ } \\
\text { predictor affecting AoSI. }\end{array}$ \\
\hline $\begin{array}{l}\text { Arida et al. } 2015 \\
\text { [11] }\end{array}$ & Cross sectional study & $\begin{array}{l}\text { Patients } n=41 \\
\text { Age: } 49 \pm 13 \text { years Female: } 88 \% \\
\text { Controls } n=41 \\
\text { Age: } 49 \pm 13 \text { years Female: } 88 \%\end{array}$ & $\begin{array}{l}\text { 75.6\% CTC } \\
75.6 \% \text { MTX } \\
7.3 \% \text { LEF } \\
41.5 \% \text { bDMARD }\end{array}$ & $\begin{array}{l}\text { No difference in arterial stiffness between RA and contro } \\
\nearrow \text { carotid and/or femoral plaques in RA compared to hea } \\
\text { controls ( } 29 \text { vs } 12 \%, p=0.039 \text { ). }\end{array}$ \\
\hline Li et al. 2013 [24] & Cross sectional study & $\begin{array}{l}\text { Patients } n=138 \\
\text { Age: } 50.2 \pm 12.5 \text { years Female: } \\
80.4 \% \\
\text { Controls } n=150 \\
\text { Age: } 49.6 \pm 8.7 \text { years Female: } \\
79.3 \%\end{array}$ & $\begin{array}{l}\text { 6.5\% sDMARD } \\
53.2 \% \text { NSAID }\end{array}$ & $\begin{array}{l}\nearrow \mathrm{PWV} \text { in RA }(1705.44 \pm 429.20 \text { vs } 1386.23 \pm \\
411.09 \mathrm{~cm} / \mathrm{s}, \mathrm{p}<0.001) . \\
\text { baPWV was independently associated with age and serun } \\
\text { triglycerides level. }\end{array}$ \\
\hline $\begin{array}{l}\text { Santos et al. } 2012 \\
\text { [53] }\end{array}$ & Cross sectional study & $\begin{array}{l}\text { RA Patients } n=107 \\
\text { Age: } 50.2 \pm 14.1 \text { years Female: } \\
\text { 100\% } \\
\text { SLE Patients } n=127 \\
\text { Age: } 43.9 \pm 13.9 \text { years Female: } \\
100 \% \\
\text { Controls } n=124 \\
\text { Age: } 46.9 \pm 13.7 \text { years Female: } \\
100 \%\end{array}$ & $\begin{array}{l}\text { RA patients: } 19 \% \text { HCQ } \\
\text { 100\% CTC } \\
82 \% \text { MTX } \\
\text { SLE patients: 74\% HCQ } \\
\text { 10.9\% CTC 10\% MTX }\end{array}$ & $\nearrow \mathrm{AIx}$ in SLE as compared to RA ( $16 \%$ vs $11 \%, \mathrm{p}=0.04$ ) \\
\hline $\begin{array}{l}\text { Pieringer et al. } 2012 \\
\text { [86] }\end{array}$ & Cross sectional study & $\begin{array}{l}\text { Patients } n=203 \\
\text { Age: } 56.3 \pm 12.2 \text { years Female: } \\
83.7 \% \\
\text { Controls } n=208 \\
\text { Age: } 49.6 \pm 12.7 \text { years Female: }\end{array}$ & $\begin{array}{l}5.9 \% \text { SLZ } \\
31.5 \% \text { CTC } \\
71.4 \% \text { MTX } \\
11.4 \% \text { LEF } \\
42 \% \text { bDMARD }\end{array}$ & $\nearrow$ AIx in RA $(30.5 \pm 9.0 \%$ vs $24.0 \pm 11.0 \%, P<0.001)$ \\
\hline
\end{tabular}

Tam et al. 2013 [87] Cohort study

Kim et al. 2012 [88] Cohort study

Ilter et al. 2016 [89] Cross sectional study

Ntusi et al. 2019 [36]

Cross sectional study

\section{Dregan 2018 [18]}

Pieringer et al. 2017 [21]

Yang et al. 2019 [14]

Byram et al. 2018

[90]

Cross sectional study

Cross sectional study

Cross sectional study
$81.7 \%$

Patients $n=95 \quad 32 \%$ SLZ

Age: $52 \pm 11$ years Female: $87 \% \quad 27 \%$ HCQ

$48 \%$ MTX

Patients $n=262$

Age: 56.7 years Female: $82 \%$

Age: $59 \pm 7$ years Female: $70 \%$

Controls $n=165,149$

Age: $57 \pm 8$ years Female: $55 \%$

Patients $n=287$

Age: $\mathbf{5 8 . 4} \pm 12.6$ years Female: $80.8 \%$

Controls $n=232$

Age: $49.9 \pm 13.4$ years Female: $80.2 \%$

Patients $n=72$

Age: $47.4 \pm 6.88$ years Female: $81.9 \%$

Controls $n=55$

Age: $46.2 \pm 6.72$ years Female:

81.8\%

Patients with RA n $=165$ Females: $69 \%$

- RA exercisers $n=63$ Age: 54 years [44-66]

- RA non exercisers $n=102$ Age: 54 years [46-61]
$9 \%$ LEF

$56.9 \%$ CTC

$84 \%$ MTX

$31.4 \%$ NSAID
$62.8 \%$ CTC

$42.8 \%$ anti-TNF $\alpha$

91\% sDMARD

$90 \%$ MTX

$60 \%$ HCQ

$25 \%$ SLZ

$16 \%$ LEF

$4 \%$ RTX $12 \%$ CTC

$36 \%$ NSAID

$13 \%$ CTC

$42 \%$ SDMARD

$56.4 \%$ achieved disease remission at 12 months. Significant reductions in AIx ( $30.7 \pm 10.5$ at baseline vs $26.3 \pm 11.1 \% p=$ 0.001 ) and PWV (for the right side $14.94 \pm 3.37$ at baseline vs $14.47 \pm 3.27 \mathrm{~m} / \mathrm{s}, p=0.017)$.

baPWV was significantly correlated with systolic blood pressure $(r=0.739, \mathrm{p}<0.001)$, age $(r=0.661, \mathrm{p}<0.001)$ but not with swollen joint count, evaluator's global assessment, CRP, and ESR.

$\nearrow \mathrm{PWV}$ in RA ( $28.3 \pm 10.3$ vs. $21.8 \pm 9.3 \mathrm{~m} / \mathrm{s}, p=0.03)$.

$\nearrow$ AIx in RA (10.2 \pm 2.3 vs. $9.2 \pm 1 \%, \mathrm{p}=0.01)$.

$70.7 \%$ MTX

$8 \%$ HCQ

$6.6 \%$ SLZ

$11.2 \% \mathrm{LEF}$

$2.4 \%$ RTX

$1.7 \%$ TCZ

$30 \%$ anti-TNF $\alpha$

$3.1 \%$ ABT

Patients had not used any CTC, IS or NSAID before the study.

MD

PWV in RA with CVRFs compared with RA without CVRFs, controls with CVRFs and controls without CVRFs ( $8.2 \pm 3.3$ vs $6.8 \pm 2.7$ vs $6.0 \pm 1.7$ vs $4.6 \pm 2.2$ respectively, $\mathrm{p}<0.001$ ).

\author{
AoSI in RA (9.47, CI95\% [9.33-9.62] vs 9.32 \\ CI95\% [9.31-9.34]).
}

AIx in RA patients $(31.1 \pm 9.3$ vs $23.6 \pm 11.3 \%, p<0.0001)$ In RA patients AIx and central PP, are correlated with SCORE and QRISK2.

In RA patients, the hfPWV $(5.49 \pm 1.19 \mathrm{~m} / \mathrm{s})$ and $\mathrm{cfPWV}(6.70$ $\pm 1.23 \mathrm{~m} / \mathrm{s}$ ) were significantly higher than that in controls ( $=0.0006, p=0.0001$, respectively). hcPWV, brPWV and faPWV only showed an increase trend without significance.

$\searrow$ PWV in exercisers compared to non- exercisers (8.48 CI95\% [7.15-9.70] vs. 9.07 [8.15-10.03] $\mathrm{m} / \mathrm{s}, p=0.005)$. Non-significant decrease in AIx in exercisers (28\% [21-35] vs. $32 \%$ [25-37], $p=0.10$ ). 


\begin{tabular}{|c|c|c|c|c|}
\hline Reference & Design & Population & Treatment & Outcome \\
\hline $\begin{array}{l}\text { Woodman et al. } 2019 \\
\text { [91] }\end{array}$ & Longitudinal study & $\begin{array}{l}\text { Patients } n=86 \\
\text { Age: } 64[56-69] \text { years Female: } \\
73 \%\end{array}$ & $\begin{array}{l}8.1 \% \text { NSAID } \\
34.9 \% \text { CTC } \\
65.1 \% \text { MTX }\end{array}$ & $\begin{array}{l}\text { Comparing } 5 \text { patterns of fatty acid intake: higher reported } \\
\text { intake of } \mathrm{n}-3 \text { fatty acids, palmitoleic acid and arachidonic acid } \\
\text { is associated with a lower } \operatorname{AIx}(p=0.023) \text {. }\end{array}$ \\
\hline $\begin{array}{l}\text { Novikova et al. } 2016 \\
\quad[31]\end{array}$ & Longitudinal study & $\begin{array}{l}\text { Patients } \mathrm{n}=55 \\
\text { Age: } 50.4 \pm 1.7 \text { years Female: } \\
100 \%\end{array}$ & $\begin{array}{l}100 \% \text { RTX } \\
98 \% \text { NSAID } \\
80 \% \text { CTC } \\
86 \% \text { sDMARD: } \\
\text { - } 55 \% \text { MTX } \\
\text { - } 38 \% \text { LEF } \\
\text { - } 7 \% \text { Others sDMARD }\end{array}$ & $\begin{array}{l}\searrow \text { PWV in RTX responders }(\mathrm{n}=41) \text {, after } 6 \text { months of treatment } \\
(9.9 \pm 0.9 \mathrm{~m} / \mathrm{s} \text { vs } 14.7 \pm 0.9 \mathrm{~m} / \mathrm{s}, p<0.05) \text {. } \\
\text { No significant decrease after } 6 \text { months in non- responders ( } n= \\
\text { 14). }\end{array}$ \\
\hline $\begin{array}{l}\text { Provan et al. } 2015 \\
\text { [33] }\end{array}$ & Prospective study & $\begin{array}{l}\text { Patients } n=36 \\
\text { - RTX n }=24 \\
\text { Age: } 56.9[25-72] \text { years Female: } \\
71 \% \\
\text { - ABT } n=5 \\
\text { Age: } 53.9[41-67] \text { years Female: } \\
\text { 100\% } \\
\text { - TCZ } n=7 \\
\text { Age: } 52.3[36-59] \text { years Female: } \\
86 \%\end{array}$ & & $\begin{array}{l}\searrow \text { PWV at } 3 \text { months only in the TCZ group }(-0.9 \pm 1.0 \mathrm{~m} / \mathrm{s}, p= \\
0.03) \text {. } \\
\searrow \text { PWV at } 12 \text { months in the RTX group }(7.3[6.8-7.9] \text { vs } 7.7 \\
[7.2-8.2] \mathrm{m} / \mathrm{s}, p=0.04) .\end{array}$ \\
\hline $\begin{array}{l}\text { Mäki-Petäjä et al. } \\
2012 \text { [26] }\end{array}$ & Prospective study & $\begin{array}{l}\text { Patients } n=17 \\
\text { Age: } 58 \pm 5 \text { years Female: } 65 \% \\
\text { Controls } n=34 \\
\text { Age: } 58 \pm 5 \text { years Female: MD }\end{array}$ & $\begin{array}{l}100 \% \text { anti-TNF } \alpha \\
-70 \% \text { ADA } \\
-30 \% \text { ETN } \\
47 \% \text { HCQ } \\
53 \% \text { MTX } \\
47 \% \text { LEF } \\
35 \% \text { CTC } \\
12 \% \text { SLZ }\end{array}$ & $\begin{array}{l}\searrow \text { PWV after } 8 \text { weeks of Anti-TNF } \alpha \text { (from } 9.09 \pm 1.77 \text { to } 8.63 \pm \\
1.42 \mathrm{~m} / \mathrm{s}, \mathrm{p}=0.04 \text { ). } \\
\searrow \text { Disease activity score after } 8 \text { weeks of Anti- TNF } \alpha \text { (from } 6.52 \\
\pm 0.78 \text { to } 4.38 \pm 1.61, \mathrm{p}<0.0001 \text { ). }\end{array}$ \\
\hline Tam et al. 2012 [27] & $\begin{array}{l}\text { Randomized opel- } \\
\text { label study }\end{array}$ & $\begin{array}{l}\text { Patients } n=40 \\
\text { - MTX + IFX } n=20 \\
\text { Age: } 53 \text { [47-61] years Female: } \\
95 \% \\
\text { - MTX n }=20 \\
\text { Age: } 53 \text { [43-59] years Female: } \\
75 \%\end{array}$ & $\begin{array}{l}\text { Among all patients: } 93 \% \\
\text { NSAID } \\
3 \% \text { LEF } \\
5 \% \text { HCQ } \\
23 \% \text { CTC }\end{array}$ & $\begin{array}{l}\text { Greater reduction in PWV in the MTX -IFX group compared with } \\
\text { the MTX alone group at } 6 \text { months }(-0.78 \pm 1.13 \mathrm{~m} / \mathrm{s} \text { vs } 0.18 \pm \\
1.59 \mathrm{~m} / \mathrm{s}, p=0.044) \text {. } \\
\text { No differences between the two groups in the changes in AIx } \\
\text { after } 6 \text { months. }\end{array}$ \\
\hline $\begin{array}{l}\text { Angel et al. } 2012 \\
\quad[28]\end{array}$ & Prospective study & $\begin{array}{l}\text { Patients } n=55(25 \text { RA, } 19 \text { AnS and } \\
11 \text { PsA }) \\
\text { - Anti-TNF- } \alpha \\
\text { Age: } 47.2 \pm 11.6 \text { years Female: } \\
39 \% \\
\text { - Controls: } \\
\text { Age: } 51.9 \pm 14.5 \text { years Female: } \\
42 \%\end{array}$ & $\begin{array}{l}\text { Among all patients: } 40 \% \\
\text { CTC } \\
44 \% \text { NSAID } \\
42 \% \text { MTX } \\
13 \% \text { SLZ }\end{array}$ & $\begin{array}{l}\text { PWV was improved in the Anti-TNF } \alpha \text { group, but not in the } \\
\text { control group }(-0.54 \pm 0.79 \mathrm{~m} / \mathrm{s} \text { vs. } 0.06 \pm 0.61 \mathrm{~m} / \mathrm{s}, p= \\
0.004) \text {. }\end{array}$ \\
\hline $\begin{array}{l}\text { Mathieu et al. } 2012 \\
\text { [92] }\end{array}$ & Prospective study & $\begin{array}{l}\text { Patients } n=33 \\
\text { Age: } 60.9 \pm 12 \text { years Female: } 88 \%\end{array}$ & $\begin{array}{l}100 \% \text { RTX } \\
60.6 \% \text { MTX } \\
3 \% \text { LEF } \\
9.1 \% \text { HCQ } \\
6.1 \% \text { Several sDMARD }\end{array}$ & $\begin{array}{l}\text { No change in PWV, at } 6 \text { or } 12 \text { months of RTX }(8.1 \pm 3.1 \text { at } \\
\text { baseline, vs } 8.1 \pm 2.8 \text { and } 8.0 \pm 2.7 \mathrm{~m} / \mathrm{s}, p=0.924) \\
\text { No change in AIx, at } 6 \text { or } 12 \text { months of RTX }(30.4 \pm 8.2 \text { at } \\
\text { baseline, vs } 28.6 \pm 7.6 \text { and } 29.4 \pm 6.7 \%, p=0.216)\end{array}$ \\
\hline $\begin{array}{l}\text { Kume et al. } 2011 \\
\text { [32] }\end{array}$ & $\begin{array}{l}\text { Open-label } \\
\text { randomized } \\
\text { controlled trial }\end{array}$ & $\begin{array}{l}\text { Patients } n=64 \\
\text { - TCZ } n=22 \\
\text { Age: } 62 \pm 16 \text { years Female: } 86 \%\end{array}$ & $\begin{array}{l}\text { No prior treatment with } \\
\text { MTX, CTC, or bDAMRD. }\end{array}$ & $\begin{array}{l}\searrow \text { CAVI at } 24 \text { weeks of treatment for all the groups (TCZ: } 0.85 \\
\pm 0.15 \mathrm{~m} / \mathrm{s}, \mathrm{p}=0.02 \text {; ETN: } 0.81 \pm 0.18 \mathrm{~m} / \mathrm{s}, \mathrm{p}=0.03 \text {; ADA: } \\
0.90 \pm 0.21 \mathrm{~m} / \mathrm{s}, \mathrm{p}=0.02 \text { ). } \\
\searrow \text { AIx at } 24 \text { weeks of treatment for all the groups (TCZ } 3.59 \pm \\
0.33 \%, \mathrm{p}=0.03 \text {; ETN } 1.03 \pm 0.44 \%, \mathrm{p}=0.03 ; \text { ADA } 3.54 \pm \\
0.52 \%, \mathrm{p}=0.02 \text { ) } \\
\triangle \mathrm{CAVI} \text { and } \triangle \mathrm{AI} \text { was not significantly different among groups. }\end{array}$ \\
\hline
\end{tabular}

Age: $61 \pm 15$ years Female: $86 \%$

- ADA $n=21$

Age: $63 \pm 17$ years Female: $86 \%$

Mäki-Petäjä et al. Cross sectional study 2006 [25]
Patients $n=77$

Age: $57 \pm 13$ years Female: $84 \%$ Controls $n=142$

Age: $57 \pm 11$ years Female: $73 \%$

Prospective study
Patients $n=9$
Age: $54 \pm 19$ years Female: MD
$17 \%$ anti-TNF $\alpha$ $58 \%$ MTX $36 \%$ Other sDMARD $74 \%$ NSAID $39 \%$ CTC $100 \%$ ETN $67 \%$ CTC $\nearrow \mathrm{PWV}$ in RA patients (8.35 [7.14-10.24] vs 7.52 [6.56-9.18] $\mathrm{m} / \mathrm{s}, \mathrm{p}=0.005$ ). No difference in AIx. 


\begin{tabular}{|c|c|c|c|c|}
\hline Reference & Design & Population & Treatment & Outcome \\
\hline & & $\begin{array}{l}\text { Controls } n=24 \\
\text { Age: MD Female: MD } \%\end{array}$ & $\begin{array}{l}33 \% \text { MTX } \\
11 \% \text { SLZ } \\
56 \% \text { NSAID }\end{array}$ & $\begin{array}{l}\searrow \text { PWV by ETN (from } 8.82 \pm 20.04 \mathrm{~m} / \mathrm{s} \text { at baseline to } 7.94 \pm \\
1.86 \text { and } 7.68 \pm 1.56 \mathrm{~m} / \mathrm{s} \text { at } 4 \text { and } 12 \text { weeks, } p=0.0003 \text { ). No } \\
\text { change in AIx. }\end{array}$ \\
\hline $\begin{array}{l}\text { Gkaliagkousi et al. } \\
2018 \text { [93] }\end{array}$ & Cross sectional study & $\begin{array}{l}\text { Patients } n=91 \\
\text { Age: } 61.4 \pm 11.9 \text { years Female: } \\
79.1 \% \\
\text { Controls } n=50 \\
\text { Age: } 58.5 \pm 7.2 \text { years Female: } 78 \%\end{array}$ & $\begin{array}{l}\text { 73.6\% SDMARD } \\
38.5 \% \text { bDMARD } \\
28.6 \% \text { CTC }\end{array}$ & $\begin{array}{l}\nearrow \text { PWV in RA }(8.3 \pm 2.2 \text { vs } 7.5 \pm 1.3 \mathrm{~m} / \mathrm{s}, p=0.01) . \\
\text { Dipping levels }(\%) \text { were inversely associated with PWV }(r= \\
-0.218, p=0.045) .\end{array}$ \\
\hline Botta et al. 2016 [94] & Cross sectional study & $\begin{array}{l}\text { Active RA } n=27 \\
\text { Age: } 56 \pm 9 \text { years Female: } 93 \% \\
\text { Inactive RA } n=17 \\
\text { Age: } 56 \pm 12 \text { years Female: } 82 \% \\
\text { Controls } n=33 \\
\text { Age: } 49 \pm 11 \text { years Female: } 79 \%\end{array}$ & $\begin{array}{l}\text { 84\% MTX } \\
18 \% \text { Anti-TNFo } \\
30 \% \text { HCQ } \\
9 \% \text { LEF } \\
2 \% \text { RTX }\end{array}$ & $\begin{array}{l}\nearrow \mathrm{PWV} \text { in active RA compared with inactive } \\
\mathrm{RA} \text { and controls }(p=0.030)\end{array}$ \\
\hline $\begin{array}{l}\text { Vassilopoulos et al. } \\
2015 \text { [29] }\end{array}$ & Prospective study & $\begin{array}{l}\text { RA sDMARD }+ \text { ADA } n=18 \\
\text { Age: } 59 \pm 13 \text { years Female: } 89 \% \\
\text { RA with MTX only } n=18 \\
\text { Age: } 61 \pm 10 \text { years Female: } 83 \%\end{array}$ & $\begin{array}{l}\text { ADA group: } \\
39 \% \text { ADA only } \\
33 \% \text { MTX } \\
24 \% \text { LEF } \\
6 \% \text { HCQ }\end{array}$ & $\begin{array}{l}\text { At } 12 \text { weeks: } \\
\text { - } \searrow \text { PWV in the ADA group (from } 8.18 \pm 2.03 \\
\text { to } 7.01 \pm 1.78 \mathrm{~m} / \mathrm{s}, p=0.00006 \text { ) }\end{array}$ \\
\hline & & & 61\% CTC & $\begin{array}{l}\text { - No significant change in PWV in the MTX group. } \\
\text { - No significant change in AIx in both groups. }\end{array}$ \\
\hline $\begin{array}{l}\text { Daïen et al. } 2013 \\
\text { [95] }\end{array}$ & Prospective study & $\begin{array}{l}\text { RA with ETN } \mathrm{n}=28 \\
\text { Age: } 57 \pm 13 \text { years Female: } 100 \% \\
\text { RA with sDMARD } n=20 \\
\text { Age: } 51 \pm 17 \text { years Female: } 100 \%\end{array}$ & $\begin{array}{l}\text { sDMARD group: } 30 \% \\
\text { CTC } \\
\text { ETN group: } 72 \% \text { CTC } \\
89 \% \text { sDMARD }\end{array}$ & $\begin{array}{l}\text { With } 6 \text { months of treatment, no significant change in PWV or } \\
\text { AIx in both groups. }\end{array}$ \\
\hline $\begin{array}{l}\text { Angel et al. } 2011 \\
\text { [96] }\end{array}$ & Prospective study & $\begin{array}{l}\text { Patients } \mathrm{n}=17(\mathrm{RA} \mathrm{n}=8 / \text { AS } \mathrm{n}= \\
6 / \text { PsA } \mathrm{n}=3) \text { Age: } 54 \pm 12.1 \text { years } \\
\text { Female: } 52.9 \%\end{array}$ & $\begin{array}{l}100 \% \text { IFX } \\
29.4 \% \text { CTC } \\
29.4 \% \text { NSAID } \\
58.8 \% \text { MTX }\end{array}$ & $\begin{array}{l}\text { No changes in AIx or PWV during the period between the two } \\
\text { IFX infusions (weeks 4-8). }\end{array}$ \\
\hline
\end{tabular}

ADA: Adalimumab; AIx: Augmentation Index; Anti-TNF $:$ TNF- $\alpha$ Inhibitors; AoSI: Aortic Stiffness Index; AS: Ankylosing Spondylitis; AZA: Azathioprine; CAVI: CardioAnkle Vascular Index; CDAI: Clinical Disease Activity Index; CRP: C-Reactive Protein; CTC: Corticoids; CVD: Cardio-Vascular Disease; sDMARD: synthetic Disease Modifying Anti-Rheumatic Drug; bDMARD: biologic Disease Modifying Anti-Rheumatic Drug; ESR: Erythrocyte Sedimentation Rate; ETN: Etanercept; HAQ-II: Health Assessment Questionnaire; HCQ: Hydroxychloroquine; HT: Hypertension; (c)IMT: (carotid) Intima-Media Thickness; IFX: Infliximab; IS: Immunosuppressive drug; IM: Immunomodulatory drug; LEF: Leflunomide; MD: Missing Data; MTX: Methotrexate; NSAID: Nonsteroidal Anti-Inflammatory Drug; PP: Pulse Pressure; PsA: Psoriasic Arthritis; PWV: Pulse Wave Velocity (hf: heart-femoral, cf.: carotid-femoral, hc: heart-carotid, br: brachial-radial, fa: femoral-ankle); QRISK2: Cardiovascular Risk Score; RA: Rheumatoid Arthritis; RF: Rheumatoid Factor; RTX: Rituximab; SCORE: Systematic Coronary Risk Estimation; SLE: Systemic Lupus Erythematosus; SLZ: Sulfasalazine; TCZ: Tocilizumab.

showed that each year of disease duration had a greater impact on the carotid to femoral PWV than age.

Second, arterial stiffness has been associated with disease activity and inflammatory markers [1]. Comparing $70 \mathrm{RA}$ patients (36 with active disease and 34 with inactive disease) to 35 controls, carotid PWV was significantly higher in patients with active RA (defined as a Disease Activity Score (DAS28) score $\geq 3.2$ ) [16]. In another study of 50 RA patients, corrected aortic AIx was correlated with Clinical Disease Activity Index (CDAI) and Health Assessment Questionnaire (HAQ-II) [17]. In a very large sample (the UK Biobank study), the association appeared to increase progressively with the severity of chronic inflammation, as assessed by a composite measure of inflammatory biomarkers [18]. We found only one study specifically exploring the correlation between the presence of rheumatoid factor (RF) and anti-cyclic citrullinated peptide (anti-CCP), and arterial stiffness. Seronegative patients had significantly higher PWV than seropositive patients $(7.68 \pm 2.24$ vs $7.12 \pm 1.34 \mathrm{~m} / \mathrm{s}$, respectively, $p=0.031$ ) [19]. However, seronegative patients had slightly higher Body Mass Index (BMI), systolic blood pressure and Creactive protein (CRP) level. Further studies are needed.

In addition, Cioffi et al. reported that Aortic Stiffness Index (AoSI) was significantly higher (almost twice as high) in RA patients than in controls matched for CV risk factors $(6.3+/-4.5$ vs. $4.6+/-3.5 \%, p<$ 0.001) [20], highlighting that increased aortic stiffening exists beyond $\mathrm{CV}$ risk factors, probably related to the inflammation itself. It also suggests that CV risk cannot be assessed solely by scores that take into account CV risk factors alone [21].

However, the influence of traditional CV risk factors must not be overlooked. Most published evidence supports that arterial stiffness in RA is also associated with age and hypertension. For example, in 149 RA patients, mean blood pressure accounted for $28.9 \%$ of the variance in PWV [22]. Besides, arterial stiffness index and common carotid intimamedia thickness have been reported to be higher in hypertensive RA patients than in hypertensive patients without RA. [23]. Brachial-anckle PWV has also been independently associated with serum triglycerides levels in RA [24]. These results emphasize that CV risk factors must be strictly controlled in RA patients as they increase the risk associated with RA itself.

\subsubsection{Influence of RA treatment on arterial stiffness}

Exploring the impact of RA treatments on arterial stiffness seems challenging given the indications selection bias and potential confounding factors. For example, in a group of 104 RA patients, $27 \%$ of whom received glucocorticoids (GC), GC use was independently associated with PWV [12]. However, patients with more severe arthritis are more likely to receive GC, which may artificially lead to increased vascular dysfunction while also reducing disease activity, which could reduce arterial stiffness.

TNF- $\alpha$ inhibitors (TNFi) seem to have a beneficial impact on arterial stiffness [25-29], as reported in a meta-analysis [30]. Improvement in carotid-femoral PWV was found independently of the reduction in DAS28. It may be assumed that RA patients with increased aortic stiffness may benefit from early prescription of TNFi [30]. A preferential effect of etanercept and adalimumab was evidenced over infliximab. Although data are more scarce, rituximab [31] and tocilizumab [32,33] have also shown a beneficial effect on arterial stiffness [34]. Thus, the reduction of AIx and PWV by biological therapy supports the role of inflammation in increasing arterial stiffness in RA. 
Table 2

Systemic Lupus Erythematosus and arterial stiffness.

\begin{tabular}{|c|c|c|c|c|}
\hline Reference & Design & Population & Treatment & Outcome \\
\hline $\begin{array}{l}\text { Montalbán-Méndez } \\
\text { et al. } 2018 \text { [42] }\end{array}$ & Cohort study & $\begin{array}{l}\text { Patients } n=49 \\
\text { Age: } 41.3 \pm 13.8 \text { years } \\
\text { Female: } 100 \%\end{array}$ & $\begin{array}{l}\text { No bDMARD or CTC } \\
>10 \mathrm{mg} / \mathrm{j} \text { in the past } 6 \\
\text { months }\end{array}$ & $\begin{array}{l}\text { CRF was significantly associated with PWV } \\
\text { - Siconolfi }(b=0.773, p<0.001) \\
\text { - 6MWT }(b=0.590, p<0.001)\end{array}$ \\
\hline $\begin{array}{l}\text { Cypiene et al. } 2017 \\
\text { [22] }\end{array}$ & $\begin{array}{l}\text { Cross sectional } \\
\text { study }\end{array}$ & $\begin{array}{l}\text { Patients } n=63 \\
\text { Age: } 53.3 \pm 15.3 \text { years } \\
\text { Female: } 87.3 \% \\
\text { Controls } n=156 \\
\text { Age: } 42.8 \pm 11.0 \text { years } \\
\text { Female: } 73.1 \%\end{array}$ & $\begin{array}{l}47.3 \% \text { HCQ } \\
2.2 \% \text { CTC } \\
39.8 \% \text { MTX } \\
58.1 \% \text { AZA }\end{array}$ & $\begin{array}{l}\text { No difference in PWV between SLE and controls. } \\
\nearrow \text { AIx in SLE ( } 25.49 \text { CI95\% [23.43-27.54] vs } 17.55 \text { CI95\% [15.97-19.12], } \\
<0.0001] \text {. } \\
\text { MBP was the only significant variable in the model and accounted for } 14.3 \% \\
\text { of the variance of PWV. }\end{array}$ \\
\hline $\begin{array}{l}\text { Tziomalos et al. } \\
2017[46]\end{array}$ & $\begin{array}{l}\text { Cross sectional } \\
\text { study }\end{array}$ & $\begin{array}{l}\text { Patients } n=55 \\
\text { Age: } 53.3 \pm 15.3 \text { years } \\
\text { Female: } 87.3 \% \\
\text { Controls } n=61 \\
\text { Age: } 55.4 \pm 8.1 \text { years } \\
\text { Female: } 91.8 \%\end{array}$ & $\mathrm{MD}$ & $\begin{array}{l}\text { After adjustment for confounders, PWV and AIx did not differ between SLE } \\
\text { and controls ( } \mathrm{B}=0.542, p=0.107 \text { and } \mathrm{B}=0.446, p=0.319 \text {, respectively). }\end{array}$ \\
\hline $\begin{array}{l}\text { Karp et al. } 2016 \\
\quad \text { [51] }\end{array}$ & $\begin{array}{l}\text { Cross sectional } \\
\text { study }\end{array}$ & $\begin{array}{l}\text { Patients } n=30 \\
\text { Age: } 41.2 \pm 13.3 \text { years } \\
\text { Female: } 100 \% \\
\text { Controls } n=53 \\
\text { Age: } 53.5 \pm 13.9 \text { years } \\
\text { Female: } 100 \%\end{array}$ & $\begin{array}{l}87 \% \text { HCQ } \\
67 \% \text { CTC } \\
20 \% \text { AZA } \\
3 \% \text { Belimumab } \\
7 \% \text { MMF }\end{array}$ & $\begin{array}{l}\text { TPWV in SLE }(6.2 \pm 2.3 \text { vs. } 5.4 \pm 1.7 \mathrm{~m} / \mathrm{s}, \mathrm{p}=0.04) \\
\text { PWV was associated with SLE }(\mathrm{B}=0.24, p=0.02) \text {, age }(\mathrm{B}=0.53, \mathrm{p}<0.001) \text {, } \\
\text { triglycerides level }(\mathrm{B}=0.31, p=0.01), \mathrm{HDL}(\mathrm{B}=0.24, \mathrm{p}=0.04) \text { and } \\
\text { duration of SLE }(\mathrm{B}=0.55, \mathrm{p}=0.01)\end{array}$ \\
\hline $\begin{array}{l}\text { Jurcut et al. } 2012 \\
\text { [55] }\end{array}$ & $\begin{array}{l}\text { Cross sectional } \\
\text { study }\end{array}$ & $\begin{array}{l}\text { SLE }+ \text { SAPL } n=12 \\
\text { Age: } 44.7 \pm 10.6 \text { years } \\
\text { Female: } 75 \% \\
\text { SLE non-SAPL } n=20 \\
\text { Age: } 44.2 \pm 16.7 \text { years } \\
\text { Female: } 85 \%\end{array}$ & $\begin{array}{l}\text { In SLE + SAPL: } \\
\text {-CTC: } 12.29 \pm 10.8 / \text { day } \\
\text {-HCQ: } 333.3 \pm 98.4 \mathrm{mg} / \\
\text { day } \\
\text { In SLE: } \\
\text {-CTC: } 18.7 \pm 12.9 \mathrm{mg} / \text { day } \\
\text {-HCQ: } 290 \pm 120.9 \mathrm{mg} / \\
\text { day }\end{array}$ & $\begin{array}{l}\nearrow \text { PWV in SLE }+ \text { SAPL patients }(6.42 \pm 0.92 \text { vs } 5.35 \pm 0.74 \mathrm{~m} / \mathrm{s}, p<0.01) \text {. } \\
\text { No difference concerning AIx. }\end{array}$ \\
\hline $\begin{array}{l}\text { Valero-Gonzalez } \\
\text { et al. } 2014 \text { [45] }\end{array}$ & Cohort study & $\begin{array}{l}\text { Patients } n=46 \\
\text { Age: } 48 \text { years Female: } \\
100 \%\end{array}$ & $\begin{array}{l}30.4 \% \text { HCQ only } 47.8 \% \\
\text { HCQ + IS } 4.3 \% \text { IS only }\end{array}$ & $\begin{array}{l}\text { PWV was strongly associated with metabolic syndrome (OR } 6.6 \text { CI95\% } \\
{[1.2-38], p<0.03 \text { ) and SLICC/ACR score (OR } 1.5 \text { CI95\%[1-2.32, } p<0.05 \text { ). }}\end{array}$ \\
\hline $\begin{array}{l}\text { Sacre et al. } 2014 \\
\text { [52] }\end{array}$ & $\begin{array}{l}\text { Cross sectional } \\
\text { controlled study }\end{array}$ & $\begin{array}{l}\text { Patients } \mathrm{n}=41 \\
\text { Age: } 39 \pm 10 \text { years } \\
\text { Female: } 83 \% \\
\text { Controls } n=36 \\
\text { Age: } 37 \pm 8 \text { years } \\
\text { Female: } 80 \%\end{array}$ & $\begin{array}{l}100 \% \text { HCQ } \\
65.9 \% \text { CTC } \\
68.3 \% \text { IS or IM } \\
\text { drugs }\end{array}$ & $\begin{array}{l}\nearrow \mathrm{PWV} \text { in SLE patients }(7.16 \pm 1.6 \text { vs } 6.36 \pm 0.8 \mathrm{~m} / \mathrm{s}, \mathrm{p}=0.01 \text { after } \\
\text { Framingham score adjustment). } \\
\nearrow \text { ICWT in SLE patients (I } 1.3160 .67 \text { vs } 0.9360 .25 \mathrm{~mm}, p=0.003 \text { after } \\
\text { Framingham score adjustment) }\end{array}$ \\
\hline $\begin{array}{l}\text { Morreale et al. } 2016 \\
\text { [97] }\end{array}$ & $\begin{array}{l}\text { Cross sectional } \\
\text { study }\end{array}$ & $\begin{array}{l}\text { Patients } \mathrm{n}=50 \\
\text { Age: } 38 \pm 11.8 \text { years } \\
\text { Female: } 90 \% \\
\text { Controls with } \\
\text { hypertension } n=50 \\
\text { Age: } 38.9 \pm 10.6 \text { years } \\
\text { Female: } 90 \%\end{array}$ & $\begin{array}{l}54 \% \text { CTC } \\
40 \% \text { AZA or MMF }\end{array}$ & $\begin{array}{l}\text { PWV was not significantly different in patients with SLE compared with } \\
\text { patients having hypertension }(8.8 \pm 2 \text { vs } 9.2 \pm 2 \mathrm{~m} / \mathrm{s}, \mathrm{p}=0.31)\end{array}$ \\
\hline $\begin{array}{l}\text { Ding et al. } 2016 \\
\text { [49] }\end{array}$ & $\begin{array}{l}\text { Cross sectional } \\
\text { study }\end{array}$ & $\begin{array}{l}\text { Patients } n=161 \\
\text { Age: } 33 \text { years Female: } \\
\text { 100\% } \\
\text { Controls } n=135 \\
\text { Age: } 32 \text { years Female: } \\
\text { 100\% }\end{array}$ & $\begin{array}{l}79.7 \% \text { HCQ } 25.8 \% \text { MMF } \\
\text { Cumulative dose of } \\
\text { prednisone on } 24 \text { months } \\
4.6 \mathrm{~g} . \\
49.7 \% \text { CYC }\end{array}$ & $\begin{array}{l}\text { The correlation coefficients between age and arterial stiffness significantly } \\
\text { differed among the SLE patients relative to controls ( } 0.46478 \text { and } 0.52612 \text {, } \\
\text { respectively; } t=2.05 ; p=0.0409 \text { ). }\end{array}$ \\
\hline $\begin{array}{l}\text { Mercurio et al. } 2019 \\
\text { [54] }\end{array}$ & $\begin{array}{l}\text { Cross sectional } \\
\text { study }\end{array}$ & $\begin{array}{l}\text { Patients } n=43 \\
\text { Age: } 41 \pm 11 \text { years } \\
\text { Female: } 9.7 \% \\
\text { Controls } n=43 \\
\text { Age: } 39 \pm 12 \text { years } \\
\text { Female: } 90.7 \%\end{array}$ & $90.7 \%$ CTC & $\begin{array}{l}\nearrow \text { AIx in SLE patient compared to controls (21.0 IQR [17] vs. } 9.0 \text { IQR [16], } p \\
<0.001) .\end{array}$ \\
\hline $\begin{array}{l}\text { Parra et al. } 2019 \\
\text { [50] }\end{array}$ & & $\begin{array}{l}\text { Patients } n=69 \\
\text { Age: } 49 \pm 16.8 \text { years } \\
\text { Female: } 100 \% \\
\text { Controls } n=34 \\
\text { Age: } 48.7 \pm 13.2 \text { years } \\
\text { Female: } 100 \%\end{array}$ & $32 \% \mathrm{HCQ}$ & $\begin{array}{l}\nearrow \text { AIx in SLE patients }(20.30 \pm 21.54 \% \text { versus } 10.84 \pm 11.51 \%, p=0.0021) \\
\nearrow \text { IMT in SLE }(0.702 \pm 0.147 \mathrm{~mm} \text { versus } 0.633 \pm 0.891 \mathrm{~mm}, p=0.012)\end{array}$ \\
\hline $\begin{array}{l}\text { Stortz et al. } 2020 \\
\text { [47] }\end{array}$ & $\begin{array}{l}\text { Cross sectional } \\
\text { study }\end{array}$ & $\begin{array}{l}\text { Patients } n=125 \\
\text { Age: } 46 \pm(35-54) \\
\text { years Female: } 88 \% \\
\text { Controls } n=104 \\
\text { Age: } 49 \text { ( } 37-56) \text { years } \\
\text { Female: } 88.7 \%\end{array}$ & $\begin{array}{l}60.8 \% \text { HCQ } 68 \% \text { CTC } \\
50.4 \% \text { IS } \\
28 \% \text { Belimumab }\end{array}$ & No difference in PWV between SLE and controls. \\
\hline
\end{tabular}

6MWT: 6 Minute Walking Test; AIx: Augmentation Index; AZA: Azathioprine; CRF: Cardio-Respiratory Stiffness; CTC: Corticoids; CYC: Cyclophosphamide bDMARD: biologic Disease Modifying Anti-Rheumatic Drug; HCQ: Hydroxychloroquine; (c)IMT: (carotid) Intima-Media Thickness; ICWT: internal carotid wall thickness; IS: Immunosuppressive drug; IM: Immunomodulatory drug; IQR: Interquartile Range; MBP: Mean Blood Pressure; MD: Missing Data; MTX: Methotrexate; PWV: Pulse Wave Velocity (hf: heart-femoral, cf.: carotid-femoral, hc: heart-carotid, br: brachial-radial, fa: femoral-ankle); SAPL: Antiphospholipid Syndrome; SLE: Systemic Lupus Erythematosus; SLICC/ACR: systemic lupus international collaborating clinics American College of Rheumatology Damage index. 
Table 3

Psoriasis and Psoriatic arthritis and arterial stiffness.

\begin{tabular}{|c|c|c|c|c|}
\hline Reference & Design & Population & Treatment & Outcome \\
\hline $\begin{array}{l}\text { Costa et al. } 2012 \\
\quad[62]\end{array}$ & $\begin{array}{l}\text { Case control } \\
\text { study }\end{array}$ & $\begin{array}{l}\text { Patients } \mathrm{n}=20 \\
\text { Age: } 38.6[22-53] \text { years Female: } \\
30 \% \\
\text { Controls } \mathrm{n}=20 \\
\text { Age: } 38.7[22-53] \text { years Female: } \\
30 \%\end{array}$ & $70 \%$ sDMARD & $\begin{array}{l}7 \text { PWV in PsA }(8.2 \pm 0.8 \text { vs } 6.8 \pm 1.0 \mathrm{~m} / \mathrm{s}, p<0.0001) \\
\text { PWV was associated with the duration of the arthritis }(\beta=0.011 ; p=0.013) \text {. }\end{array}$ \\
\hline $\begin{array}{l}\text { Shang et al. } 2012 \\
\text { [72] }\end{array}$ & $\begin{array}{l}\text { Cross } \\
\text { sectional } \\
\text { study }\end{array}$ & $\begin{array}{l}\text { Patients } n=73 \\
\text { Age: } 47.1 \pm 12 \text { years Female: } 49 \% \\
\text { Controls } n=50 \\
\text { Age: } 44 \pm 14 \text { years Female: } 44 \%\end{array}$ & $\begin{array}{l}64.4 \% \text { NSAID } \\
56.2 \% \text { sDMARD } \\
8.2 \% \text { CTC }\end{array}$ & $\begin{array}{l}\text { AIx was higher in PsA patients with HT or LVH than PsA patients without HT } \\
\text { or LVH than controls }(29.1 \pm 15.3 \text { vs } 22.0 \pm 15.5 \text { vs } 14.7 \pm 8.8 \%, p=0.001) \text {. } \\
\text { Positive correlation with the duration of the arthritis }(r=0.259, p=0.036) \text {. }\end{array}$ \\
\hline $\begin{array}{l}\text { Shen et al. } 2015 \\
\text { [71] }\end{array}$ & $\begin{array}{l}\text { Case control } \\
\text { study. }\end{array}$ & $\begin{array}{l}\text { Patients } n=72 \\
\text { Age: } 49.6 \pm 1.7 \text { years Female: } 50 \% \\
\text { Controls } n=47 \\
\text { Age: } 43.1 \pm 10.2 \text { years Female: } \\
72.3 \%\end{array}$ & $\begin{array}{l}45.8 \% \text { NSAID } \\
50 \% \text { sDMARD } \\
6.5 \% \text { CTC }\end{array}$ & $\begin{array}{l}\nearrow \text { PWV in PSA }(14.6 \pm 2.9 \text { vs } 13.23 \pm 3.8, p=0.008) . \\
\text { Cumulative averages of repeated measures of ESR were associated with high } \\
\text { PWV. }\end{array}$ \\
\hline $\begin{array}{l}\text { Kim et al. } 2015 \\
\quad[98]\end{array}$ & $\begin{array}{l}\text { Cross } \\
\text { sectional } \\
\text { study }\end{array}$ & $\begin{array}{l}\text { Patients } n=45 \text { PsO }+9 \text { PSA } \\
\text { Age: } 41.8 \pm 12.9 \text { years Female: } 52 \% \\
\text { Controls } n=60 \\
\text { Age: } 39.3 \pm 11.5 \text { years Female: } 53 \%\end{array}$ & $\begin{array}{l}9.3 \% \text { topical only } \\
59.3 \% \text { MTX } \\
50 \% \text { CIC } \\
59.3 \% \\
\text { Phototherapy } \\
15.5 \% \text { bDMARD }\end{array}$ & $\begin{array}{l}\text { BSI in Ps0 ( } 8.15 \pm 3.72 \text { vs } 5.85 \pm 2.05, \mathrm{p}<0.001) \\
\text { No statistical difference in cIMT. }\end{array}$ \\
\hline $\begin{array}{l}\text { Dattilo et al. } 2018 \\
\text { [67] }\end{array}$ & $\begin{array}{l}\text { Cross } \\
\text { sectional } \\
\text { study }\end{array}$ & $\begin{array}{l}\text { Patients } n=33 \\
\text { Age: } 35.6 \pm 5.7 \text { years Female: } 43 \% \\
\text { Controls } n=33 \\
\text { Age: } 36.3 \pm 5.9 \text { years Female: } 45 \%\end{array}$ & $\begin{array}{l}\text { No systemic } \\
\text { treatment }\end{array}$ & $\nearrow \mathrm{PWV}$ in $\mathrm{PsO}(9.23 \pm 1.53$ vs $8.06 \pm 1.68 \mathrm{~m} / \mathrm{s}, p<0.004)$ \\
\hline 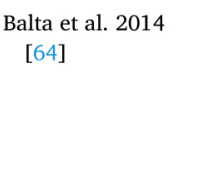 & $\begin{array}{l}\text { Cross } \\
\text { sectional } \\
\text { study }\end{array}$ & $\begin{array}{l}\text { Patients } n=32 \\
\text { Age: } 34.6+10.3 \text { years Female: } \\
100 \% \\
\text { Controls } n=35 \\
\text { Age: } 36.8+10.1 \text { years Female: } \\
100 \%\end{array}$ & No treatment & $\begin{array}{l}\nearrow \mathrm{PWV} \text { in PsO ( } 7.63 \text { vs } 6.96 \mathrm{~m} / \mathrm{s}, p=0.01) \text { PWV correlated positively with } \\
\text { age, sex, BMI, diastolic blood pressure, and CRP level }(p<0.05) \text {. }\end{array}$ \\
\hline $\begin{array}{l}\text { Altekin et al. } 2012 \\
\text { [66] }\end{array}$ & $\begin{array}{l}\text { Cross } \\
\text { sectional } \\
\text { study }\end{array}$ & $\begin{array}{l}\text { Patients } n=57 \\
\text { Age: } 41 \pm 10.8 \text { years Female: } 45.6 \% \\
\text { Controls } n=60 \\
\text { Age: } 40 \pm 9.4 \text { years Female: } 46.7 \%\end{array}$ & No treatment & $\begin{array}{l}\nearrow \mathrm{PWV} \text { in } \mathrm{PsO}(7.04 \pm 1.1 \text { vs } 6.03 \pm 0.61 \mathrm{~m} / \mathrm{s}, p<0.001) \\
\nearrow \mathrm{cIMT} \text { in } \mathrm{PsO}(0.73 \pm 0.09 \text { vs } 0.66 \pm 0.06 \mathrm{~mm}, \mathrm{p}<0.001)\end{array}$ \\
\hline $\begin{array}{l}\text { Sunbul et al. } 2015 \\
\text { [65] }\end{array}$ & $\begin{array}{l}\text { Cross } \\
\text { sectional } \\
\text { study }\end{array}$ & $\begin{array}{l}\text { Patients } n=50 \\
\text { Age: } 43.3 \pm 13.2 \text { years Female: } 48 \% \\
\text { Controls } n=50 \\
\text { Age: } 45.0 \pm 6.1 \text { years Female: } 34 \%\end{array}$ & $\begin{array}{l}34 \% \text { topical } \\
26 \% \text { oral retinoid } \\
10 \% \\
\text { phototherapy } \\
36 \% \text { MTX } \\
12 \% \text { CIC } \\
12 \% \text { ETN } \\
10 \% \text { IFX }\end{array}$ & $\begin{array}{l}\nearrow \mathrm{PWV} \text { in } \mathrm{PsO}(6.78 \pm 1.42 \text { vs } 6.18 \pm 0.80 \mathrm{~m} / \mathrm{s}, p=0.011) \\
\nearrow \mathrm{AIx} \text { in } \mathrm{PsO}(25.8 \pm 13.1 \text { vs } 17.4 \pm 12.3 \%, \mathrm{p}=0.001)\end{array}$ \\
\hline $\begin{array}{l}\text { Ikonomidis et al. } \\
2015 \text { [68] }\end{array}$ & $\begin{array}{l}\text { Cross } \\
\text { sectional } \\
\text { study }\end{array}$ & $\begin{array}{l}\text { Patients } n=59 \\
\text { Age: } 51 \pm 12.2 \text { years Female: } 37 \% \\
\text { Controls with CAD } n=59 \\
\text { Age: } 53 \pm 9 \text { years Female: } 30 \% \\
\text { Controls } n=40 \\
\text { Age: } 49 \pm 9 \text { years Female: } 45 \%\end{array}$ & $100 \%$ CIC & $\begin{array}{l}\text { PsO patients had higher PWV than healthy controls }(\mathrm{p}<0.05) \text { but similar } \\
\text { PWV than CAD patient }(p>0.05)(0.4 \pm 1.8 \text { vs } 8.6 \pm 1.5 \text { vs } 10.3 \pm 2 \mathrm{~m} / \mathrm{s}) \text {. } \\
\text { PsO patients had higher AIx than healthy controls }(\mathrm{p}<0.05) \text { but similar AIx } \\
\text { than CAD patient }(\mathrm{p}>0.05) .\end{array}$ \\
\hline $\begin{array}{l}\text { Oliveira et al. } 2019 \\
\text { [69] }\end{array}$ & $\begin{array}{l}\text { Cross } \\
\text { sectional } \\
\text { study }\end{array}$ & $\begin{array}{l}\text { Patients } n=11 \\
\text { Age: } 64 \pm 12.5 \text { years Female: } 0 \% \\
\text { Controls } n=33 \\
\text { Age: } 60 \pm 9 \text { years Female: } 0 \%\end{array}$ & $\begin{array}{l}18 \% \text { MTX } \\
9 \% \text { Topical CTC }\end{array}$ & $\begin{array}{l}\nearrow \mathrm{PWV} \text { in } \mathrm{PsO}(9.1 \pm 1.8 \text { and } 8 \pm 2 \mathrm{~m} / \mathrm{s}, p=0.033) \\
\text { No difference on } \mathrm{AIx} \text { between } \mathrm{PsO} \text { and controls. }\end{array}$ \\
\hline $\begin{array}{l}\text { Patschan et al. } \\
2018 \text { [63] }\end{array}$ & $\begin{array}{l}\text { Cross } \\
\text { sectional } \\
\text { study }\end{array}$ & $\begin{array}{l}\text { Patients with } \mathrm{PsO} n=30 \\
\text { Age: } 49 \pm 2.8 \text { years Female: } 43 \% \\
\text { Patients with PsA } n=31 \\
\text { Age: } 47.7 \pm 2.2 \text { years Female: } 48 \% \\
\text { Controls } n=26 \\
\text { Age: MD Female: MD }\end{array}$ & $\begin{array}{l}\text { PsO: } \\
33.3 \% \text { bDMARD } \\
\text { PsA: } \\
45.1 \% \text { bDMARD }\end{array}$ & No differences in AIx and PWV between PsO, PSA and controls. \\
\hline $\begin{array}{l}\text { Hansen et al. } 2018 \\
\quad[70]\end{array}$ & $\begin{array}{l}\text { Cross } \\
\text { sectional } \\
\text { study }\end{array}$ & $\begin{array}{l}\text { Patients } n=254 \\
\text { Age: } 53.9 \pm 11.9 \text { years Female: } \\
42.5 \% \\
\text { Controls } n=4431 \\
\text { Age: } 52.4 \pm 12.8 \text { years Female: } \\
47.6 \%\end{array}$ & MD & $\nearrow \mathrm{PWV}$ in $\mathrm{PsO}(8.62 \pm 2.34$ vs. $8.31 \pm 2.31 \mathrm{~m} / \mathrm{s}, p=0.04)$ \\
\hline $\begin{array}{l}\text { Di Vito et al. } 2018 \\
\quad[99]\end{array}$ & $\begin{array}{l}\text { Cross } \\
\text { sectional } \\
\text { study }\end{array}$ & $\begin{array}{l}\text { Low CV risk patients } n=34 \\
\text { Age: } 42.5 \pm 10.3 \text { years Female: } 47 \% \\
\text { Low CV risk controls } n=39 \\
\text { Age: } 46.2 \pm 6.7 \text { years Female: } 54 \% \\
\text { High CV risk patients } n=23 \\
\text { Age: } 55.7 \pm 11.3 \text { years Female: } 43 \% \\
\text { High CV risk controls } n=15 \\
\text { Age: } 57.9 \pm 6.5 \text { years Female: } 57 \%\end{array}$ & MD & $\begin{array}{l}\text { In the High CV risk group: } \\
\nearrow \text { PWV in patients with PsO }(8.7 \pm 1.6 \text { vs. } 7.5 \pm 0.9 \mathrm{~m} / \mathrm{s}, p=0.024) \text {. } \\
\text { In the Low CV risk group: } \\
\text { No difference in PWV between groups. }\end{array}$ \\
\hline
\end{tabular}


Table 3 (continued)

\begin{tabular}{|c|c|c|c|c|}
\hline Reference & Design & Population & Treatment & Outcome \\
\hline Dregan 2018 [18] & $\begin{array}{l}\text { Cross } \\
\text { sectional } \\
\text { study }\end{array}$ & $\begin{array}{l}\text { Patients } n=2091 \\
\text { Age: } 57 \pm 8 \text { years Female: } 48 \% \\
\text { Controls } n=165,149 \\
\text { Age: } 57 \pm 8 \text { years Female: } 55 \%\end{array}$ & $\begin{array}{l}15 \% \text { NSAID } \\
12 \% \text { SDMARD } \\
8 \% \text { CTC }\end{array}$ & $\nearrow \mathrm{SI}$ in Pso (9.49 IC95\%[9.36-9.61] vs 9.32 CI95\%[9.31-9.34]) \\
\hline
\end{tabular}

AIx: Augmentation Index; BSI: Beta Stiffness Index; CAD: Coronary Artery Disease; CIC: Ciclosporine; CRP: C-Reactive Protein; CTC: Corticoids; CV: Cardio- Vascular; sDMARD: synthetic Disease Modifying Anti-Rheumatic Drug; bDMARD: biologic Disease Modifying Anti-Rheumatic Drug; ESR: Erythrocyte Sedimentation Rate; ETN: Etanercept; HT: Hypertension; (c)IMT: (carotid) Intima-Media Thickness; IFX: Infliximab; LVH: Left Ventricular Hypertrophy MD: Missing Data; MTX: Methotrexate; NSAID: Nonsteroidal Anti-Inflammatory Drug; PsA: Psoriasic Arthritis; PsO: Psoriasis; SI: Stiffness Index PWV: Pulse Wave Velocity (hf: heart-femoral, cf.: carotidfemoral, hc: heart-carotid, br: brachial-radial, fa: femoral-ankle).

\subsubsection{Influence of arterial stiffness on cardiac function and atherosclerosis}

By increasing systolic blood pressure, ventricular afterload and myocardial oxygen consumption increase, and by reducing coronary perfusion, arterial stiffness lead to heart failure [8]. In this regard, RA patients have an almost two-fold higher incidence of heart failure and this is primarily associated with diastolic dysfunction [35].

In a setting of $104 \mathrm{RA}$ patients (DAS28-ESR $3.5 \pm 1.2$ ), the thoracic fluid content index, which reflects diastolic dysfunction, was significantly elevated compared to controls [12]. In another study, there was no difference in overall LV systolic function size and mass between RA patients and matched controls, whereas regional function as assessed by magnetic resonance strain imaging was impaired in RA [36].

Besides, whether arterial rigidity may be a surrogate marker for atherosclerosis in RA patients is still under debate. For example, in 163 patients, PWV was not associated with carotid plaque [37]. In a smaller cohort of 96 individuals, without clinical CVD and classical CV risk factors, the 48 patients with RA had more than twice the prevalence of carotid and/or femoral atheromatous plaques compared with healthy controls, whereas all indices assessing arterial stiffness were similar between the two groups [11]. Authors raised the fact that arterial stiffness may also induce functional changes that may contribute to $\mathrm{CV}$ risk beyond morphological changes [38]. These results suggest that arterial stiffness may contribute to CV event through adverse hemodynamic effects rather than through increased atherogenesis. Diastolic dysfunction has been documented in RA patients without CVD, suggesting that increased PWV or/and AIx may contribute to CV events in RA, also via their adverse hemodynamic effects [39].

\subsection{Lupus and arterial stiffness}

\subsubsection{Increased arterial stiffness in SLE}

The prognosis for SLE has improved considerably in recent decades, leading to new comorbidities, such as CVD, which is now the main cause of death in this population $[1,40]$. Some studies have suggested that SLE is an independent CV risk factor [41]. It has also been shown that traditional CV risk factors, such as hypertension, dyslipidemia and diabetes, do not fully explain the excess CV morbidity and mortality [42-44]. Surrogate markers, such as PWV and AIx, should be evaluated to help identify atherosclerosis at the earliest stage [45]. With the exception of two [46] [47], all studies, including a meta-analysis [48], reported an increase in arterial stiffness in SLE compared with controls. This difference is noticeable even in younger age groups [49].

\subsubsection{Influence of disease characteristics and CV risk factors on arterial stiffness}

The increase in arterial stiffness is not solely explained by CV risk factors $[47,50,51]$, which may mean that the assessment of arterial stiffness may also be useful for CV risk stratification. However, as for RA, traditional CV risk factors should not be overlooked and should be managed as early as possible. For example, in a group of 93 SLE patients, mean blood pressure accounted for $14.3 \%$ of the variance of PWV. The differences in AIx and carotid-radial PWV were greater in younger patients, when inflammation played the main role, but less significant in older patients, as age added to other influencing factors [22]. Similarly, physical activity should be enhanced. Improved cardiorespiratory fitness may reduce age-related arterial stiffness in women with SLE [42].

Regarding the characteristics of SLE affecting arterial stiffness, in several studies, there was no association between Systemic Lupus Erythematosus Disease Activity Index (SLEDAI) and arterial stiffness $[22,45,52,53]$. This could be explained by the fact that SLEDAI echoes severity at a specific point in time but does not correlate with disease burden. However, a significant association between PWV and a damage score, the Systemic Lupus Erythematosus International Collaborating Clinics (SLICC/ACR score), was reported [45,54]. In addition, intima media thickness and PWV values were significantly higher in patients having both SLE and anti- phospholipid syndrome (APLS) than in patients with SLE or APLS alone [55]. An increase in arterial stiffness was also reported to be associated with the presence of $\beta 2$-glycoprotein 1 antibodies [50].

\subsection{Sjogren's syndrome and arterial stiffness}

\subsubsection{Increased arterial stiffness in Sjogren's syndrome}

Five articles were found to explore the relationship between Sjogren's syndrome and arterial stiffness. The largest study involved 75 women with primary Sjogren's syndrome who had higher mean PWV than controls $(8.2 \pm 1.5 \mathrm{~m} / \mathrm{s}$ vs. $7.5 \pm 1.4 \mathrm{~m} / \mathrm{s} ; p=0.01)$ [56].

\subsubsection{Influence of disease characteristics and CV risk factors on arterial stiffness}

Arterial stiffness was associated with age, blood pressure and LDL levels [56], again highlighting the importance of controlling CV risk factors. Currently, there is insufficient data to conclude whether there is an association between disease activity and arterial stiffness, particularly because of small cohorts sizes and low activity levels [57]. The influence of Sjogren's characteristics on arterial stiffness needs to be studied in more detail.

\subsubsection{Influence of arterial stiffness on cardiac function}

Although cardiac involvement in patients with primary Sjogren's syndrome is less frequent than in patients with other rheumatic diseases such as RA and SLE, left ventricular diastolic dysfunction has been described [58]. This cardiac dysfunction could be linked to the increased arterial stiffness in this population. For instance, one study involving 22 patients with primary Sjogren's syndrome reported that LV radial and longitudinal strain was impaired, compared with controls and that PWV was increased [59].

\subsection{Psoriasis, psoriatic arthritis and arterial stiffness}

\subsubsection{Increased arterial stiffness in psoriasis and psoriatic arthritis}

Increased CV morbidity in patients with psoriasis (Pso) and psoriatic arthritis (PsA) is well established [60]. Although CV risk factors play a major role [61] and should be controlled as early as possible, again, they do not fully explain excess mortality, and other factors, such as arterial stiffness, should be considered in management. For example, when 
Table 4

Spondyloarthritis and arterial stiffness.

\begin{tabular}{|c|c|c|c|c|}
\hline Reference & Design & Population & Treatment & Outcome \\
\hline $\begin{array}{l}\text { Berg et al. } 2018 \\
\quad \text { [80] }\end{array}$ & $\begin{array}{l}\text { Cohort } \\
\text { study }\end{array}$ & $\begin{array}{l}\text { Patients } n= \\
118 \\
\text { Age: } 48.9 \pm \\
11.2 \text { years } \\
\text { Female: } 36 \%\end{array}$ & $\begin{array}{l}65 \% \text { NSAID } \\
20 \% \text { Anti- } \\
\text { TNF } \alpha\end{array}$ & $\begin{array}{l}\text { Inverse } \\
\text { relations } \\
\text { between } \\
\text { VO2peak and } \\
\text { both AIx ( } p< \\
0.001) \text { and PWV } \\
(\mathrm{p}<0.001) \text {. }\end{array}$ \\
\hline $\begin{array}{l}\text { Berg et al. } 2015 \\
\quad \text { [78] }\end{array}$ & $\begin{array}{l}\text { Cohort } \\
\text { study }\end{array}$ & $\begin{array}{l}\text { Patients } n=85 \\
\text { Age: } 47.3 \pm \\
\text { 12.6 years } \\
\text { Female: } \\
41.2 \%\end{array}$ & $\begin{array}{l}83.5 \% \\
\text { NSAID } \\
16.5 \% \\
\text { sDMARD } \\
1.2 \% \text { Anti- } \\
\text { TNF } \alpha \\
3.5 \% \text { CTC }\end{array}$ & $\begin{array}{l}\text { AIx increased } \\
\text { stepwise with } \\
\text { increasing CRP } \\
(p=0.01), \text { ESR } \\
(p=0.05) \text { and } \\
\text { ASDAS ( } \mathrm{p}= \\
0.04) .\end{array}$ \\
\hline $\begin{array}{l}\text { Dzieża- } \\
\text { Grudnik } \\
\text { et al. } 2017 \\
\text { [10] }\end{array}$ & $\begin{array}{l}\text { Cross } \\
\text { sectional } \\
\text { study }\end{array}$ & $\begin{array}{l}\text { Patients } n=45 \\
\text { Age: } 33 \pm 6.6 \\
\text { years Female: } \\
13.3 \% \\
\text { Controls } n= \\
29 \\
\text { Age: } 32 \pm 7.6 \\
\text { years Female: } \\
44.8 \%\end{array}$ & $\begin{array}{l}92 \% \text { NSAID } \\
5 \% \text { CTC }\end{array}$ & $\begin{array}{l}\text { No differences } \\
\text { between AS } \\
\text { patients and } \\
\text { controls: } \\
\text { - PWV (10.7 } \\
\text { CI95\% } \\
{[9.1-11.8] \mathrm{vs}} \\
9.2 \\
\text { CI95\% } \\
{[8.3-11.4] \mathrm{m} / \mathrm{s},} \\
p=0.14) \\
\text { - AIx }(5.7 \pm 12.4 \\
\text { vs } 10 \pm 12.8 \% \text {; } \\
p=0.22)\end{array}$ \\
\hline $\begin{array}{l}\text { Avram et al. } \\
2016 \text { [76] }\end{array}$ & $\begin{array}{l}\text { Cross } \\
\text { sectional } \\
\text { study }\end{array}$ & $\begin{array}{l}\text { Patients } \mathrm{n}=24 \\
\text { Age: } 45.8 \pm \\
11.7 \text { years } \\
\text { Female: } 17 \% \\
\text { Controls } n= \\
24 \\
\text { Age: } 44.9 \pm \\
12.2 \text { years } \\
\text { Female: } 17 \%\end{array}$ & $\begin{array}{l}83 \% \text { NSAID } \\
29 \% \text { SLZ } \\
50 \% \text { anti- } \\
\text { TNF } \alpha\end{array}$ & $\begin{array}{l}\nearrow \mathrm{PWV} \text { in AS } \\
(8.7 \mathrm{CI} 95 \% \\
{[7.3-10] \text { vs } 6.7} \\
\text { CI95\% } \\
{[6.2-7.2], p=} \\
0.047) . \\
\nearrow \text { AIx }(25.5 \\
\text { CI95\% } \\
{[20.6-30.5] \text { vs }} \\
17.7 \text { CI95\% } \\
{[12-23.5] \%, p} \\
=0.038) . \\
\text { PWV and AIx } \\
\text { were } \\
\text { significantly } \\
\text { associated with } \\
\text { BASFI score ( } p \\
=0.012, r= \\
0.504 ; \text { and } p= \\
0.041, r=0.421 \\
\text { respectively). }\end{array}$ \\
\hline $\begin{array}{l}\text { Berg et al. } 2015 \\
\quad \text { [74] }\end{array}$ & $\begin{array}{l}\text { Cross } \\
\text { sectional } \\
\text { study }\end{array}$ & $\begin{array}{l}\text { Patients } n= \\
151 \\
\text { Age: } 49.2 \pm \\
11.1 \text { years } \\
\text { Female:39.1\% } \\
\text { Controls } n= \\
134 \\
\text { Age: } 52.7 \pm \\
11.4 \text { years } \\
\text { Female: } 41.8 \%\end{array}$ & $\begin{array}{l}65.6 \% \\
\text { NSAID } \\
16 \% \\
\text { sDMARD } \\
19.3 \% \text { anti- } \\
\text { TNF } \alpha \\
7.9 \% \text { CTC }\end{array}$ & $\begin{array}{l}\nearrow \text { AIx in AS } \\
(19.4 \pm 0.7 \text { vs } \\
16.9 \pm 0.7, \mathrm{p}= \\
0.01) . \\
\text { No difference in } \\
\text { PWV between } \\
\text { groups. }\end{array}$ \\
\hline $\begin{array}{l}\text { Ozkaramandli } \\
\text { Gur et al. } \\
2018 \text { [82] }\end{array}$ & $\begin{array}{l}\text { Cross } \\
\text { sectional } \\
\text { study }\end{array}$ & $\begin{array}{l}\text { Patients } n=75 \\
\text { Age: } 41.7 \pm \\
\text { 10.1 years } \\
\text { Female: } 37.3 \% \\
\text { Controls } n= \\
30 \\
\text { Age: } 38.5 \pm \\
9.9 \text { years } \\
\text { Female: } 43.3 \%\end{array}$ & $\begin{array}{l}52 \% \text { NSAID } \\
25 \% \\
\text { sDMARD } \\
52 \% \text { Anti- } \\
\text { TNF } \alpha\end{array}$ & $\begin{array}{l}\nearrow \mathrm{PWV} \text { in } \mathrm{AS} \\
(5.9 \pm 1.46 \mathrm{vs} \\
5.2 \pm 0.85 \mathrm{~m} / \mathrm{s} \\
p=0.011)\end{array}$ \\
\hline $\begin{array}{l}\text { Ozen et al. } \\
2018 \text { [79] }\end{array}$ & $\begin{array}{l}\text { Cross } \\
\text { sectional } \\
\text { study }\end{array}$ & $\begin{array}{l}\text { Patients } n=55 \\
\text { Age: } 43.33 \pm \\
7.87 \text { years } \\
\text { Female: } 41.8 \% \\
\text { Controls } n= \\
20\end{array}$ & $\begin{array}{l}27 \% \text { NSAID } \\
14.7 \% \\
\text { sDMARD } \\
47 \% \text { Anti- } \\
\text { TNF } \alpha\end{array}$ & $\begin{array}{l}\nearrow \text { AoSI in AS } \\
(3.32 \pm 1.58 \text { vs } \\
2.3 \pm 0.96, p= \\
0.009)\end{array}$ \\
\hline
\end{tabular}

Table 4 (continued)

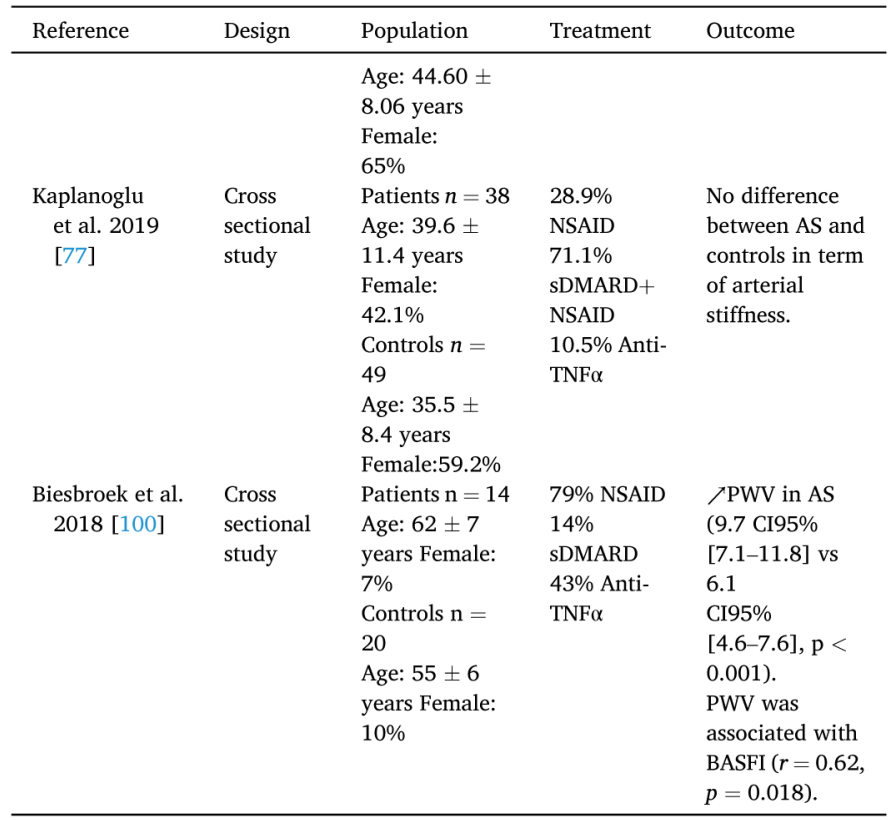

AIx: Augmentation Index; Anti-TNF $\alpha$ : TNF- $\alpha$ Inhibitors; AoSI: Aortic Stiffness Index; AS: Ankylosing Spondylitis; ASDAS: Ankylosing Spondylitis Disease Activity Score; BASFI: Bath Ankylosing Spondylitis Functionnal Index; CRP: CReactive Protein; CTC: Corticoids; sDMARD: synthetic Disease Modifying AntiRheumatic Drug; ESR: Erythrocyte Sedimentation Rate; NSAID: Nonsteroidal Anti-Inflammatory Drug; PWV: Pulse Wave Velocity (hf: heart-femoral, cf.: carotid-femoral, hc: heart-carotid, br: brachial-radial, fa: femoral-ankle); SLZ: Sulfasalazine; VO2peak: Oxygen uptake peak.

comparing 20 PsA patients to 20 controls, all free of CV risk factors, PWV was significantly higher in the PsA group [62].

With the exception of one study [63], arterial stiffness was greater in PsO and PsA patients than in controls in all the studies [64-70]. One study showed that the level of PWV in psoriatic patients was as high as that observed in patients with coronary artery disease (CAD) [68].

\subsubsection{Influence of disease characteristics and $C V$ risk factors on arterial stiffness}

Costa and al. showed that in PsA patients, increased arterial stiffness was directly associated with the duration of joint involvement rather than the duration of psoriasis itself [62].

The differing results on whether arterial stiffness in PsO and PsA is associated with disease activity and severity [71] [64] [68] [66] may be explained by the lack of reproducibility of studies.

in terms of patient age, duration, severity of the disease and treatment. Further studies are needed.

\subsubsection{Influence of arterial stiffness on cardiac function}

In a cohort of 73 PsA patients, compared to controls, PsA patients had increased ventricular and arterial stiffness and early impairment of LV longitudinal systolic and diastolic function. These results were also reported in PsO [67]. Cardiac involvement was associated with disease duration [72] [67]. LV longitudinal strain and strain rate in PsO patients were similar to those in patients with CAD [68].

\subsection{Ankylosing spondylitis and arterial stiffness}

\subsubsection{Increased arterial stiffness in ankylosing spondylitis}

Mortality in patients with ankylosing spondylitis (AnS) is 1.5 times higher than in the general population and $20 \%-40 \%$ of these deaths are thought to be due to CV events [73]. Increased arterial stiffness in this population may be detected even though the European Heart Score and 
Table 5

Sjögren's syndrome and arterial stiffness.

\begin{tabular}{|c|c|c|c|c|}
\hline Reference & Design & Population & Treatment & Outcome \\
\hline $\begin{array}{l}\text { Sabio et al. } \\
2015 \\
{[57]}\end{array}$ & $\begin{array}{l}\text { Cross } \\
\text { sectional } \\
\text { study }\end{array}$ & $\begin{array}{l}\text { Patients } \mathrm{n}= \\
44 \\
\text { Age: } 52 \\
\text { [44-56] } \\
\text { years Female: } \\
100 \% \\
\text { Controls } n= \\
78 \\
\text { Age: } 50 \\
\text { [43-55] } \\
\text { years Female: } \\
\text { 100\% }\end{array}$ & $\begin{array}{l}19 \% \text { NSAID } \\
34 \% \text { HCQ } \\
21 \% \text { CTC } \\
16 \% \text { AZA } \\
5 \% \text { MTX } \\
2 \% \text { MMF }\end{array}$ & $\begin{array}{l}\nearrow \mathrm{PWV} \text { in SS } \\
\text { patients (8.2 [IQR } \\
7.1-9.5] \text { vs } 7.7 \\
\text { [IQR 7.0-8.5] m/s } \\
\mathrm{p}=0.03) . \\
\text { Parameter } \\
\text { associated with } \\
\text { elevated PWV: } \\
\text { metabolic } \\
\text { syndrome, age, } \\
\text { SSDI and the } \\
\text { absence of SSA and } \\
\text { SSB }\end{array}$ \\
\hline $\begin{array}{l}\text { Sezis } \\
\text { Demirci } \\
\text { et al. } \\
2016 \\
\text { [56] }\end{array}$ & $\begin{array}{l}\text { Cross } \\
\text { sectional } \\
\text { study }\end{array}$ & $\begin{array}{l}\text { Patients } \mathrm{n}= \\
75 \\
\text { Age: } 54.0 \pm \\
9.3 \text { years } \\
\text { Female: } \\
100 \% \\
\text { Controls } n= \\
68 \\
\text { Age: } 51.9 \pm \\
9.9 \text { years } \\
\text { Female: } \\
100 \%\end{array}$ & $\begin{array}{l}13.7 \% \text { CTC } \\
9.6 \% \text { IS }\end{array}$ & $\begin{array}{l}\nearrow \mathrm{PWV} \text { in SS } \\
\text { patients }(8.2 \pm 1.5 \\
\text { vs. } 7.5 \pm 1.4 \mathrm{~m} / \mathrm{s} \\
\mathrm{p}=0.01)\end{array}$ \\
\hline $\begin{array}{l}\text { Zardi et al. } \\
2014 \\
{[101]}\end{array}$ & $\begin{array}{l}\text { Cross } \\
\text { sectional } \\
\text { study }\end{array}$ & $\begin{array}{l}\text { Patients } \mathrm{n}= \\
18 \\
\text { Age: } 70 \text { years } \\
\text { Female: } \\
100 \% \\
\text { Controls } \\
\text { (OA) } n=18 \\
\text { Age: } 69 \text { years } \\
\text { Female: } \\
100 \%\end{array}$ & $\begin{array}{l}\text { No specific } \\
\text { treatment. }\end{array}$ & $\begin{array}{l}\text { No difference in } \\
\text { vascular stiffness. } \\
\text { No difference in } \\
\text { carotid plaque. }\end{array}$ \\
\hline $\begin{array}{c}\text { Atzeni } \\
\text { et al. } \\
2014 \\
\text { [59] }\end{array}$ & $\begin{array}{l}\text { Cross } \\
\text { sectional } \\
\text { study }\end{array}$ & $\begin{array}{l}\text { Patients } \mathrm{n}= \\
22 \\
\text { Age: } 60.1 \pm \\
7.8 \text { years } \\
\text { Female: } 72 \% \\
\text { Controls } n= \\
22 \\
\text { Age: } 60.1 \pm \\
7.8 \text { years } \\
\text { Female: } 72 \%\end{array}$ & $\begin{array}{l}\text { The majority } \\
\text { of the patients } \\
\text { were being } \\
\text { treated with } \\
\text { HCQ, AZA and } \\
\text { MTX. } \\
32 \% \text { CTC }\end{array}$ & $\begin{array}{l}\nearrow \mathrm{PWV} \text { in SS } \\
\text { patients }(8.90 \\
{[8.00-10.00] \text { vs }} \\
6.89[6.30-7.10] \\
\mathrm{m} / \mathrm{s}, p<0.0001) . \\
\text { No difference in } \\
\text { CIMT. }\end{array}$ \\
\hline $\begin{array}{l}\text { Ozisler } \\
\text { et al. } \\
2019 \\
\text { [102] }\end{array}$ & $\begin{array}{l}\text { Cross } \\
\text { sectional } \\
\text { study }\end{array}$ & $\begin{array}{l}\text { Patients } \mathrm{n}= \\
30 \\
\text { Age: } 41.4 \pm \\
9.4 \text { years } \\
\text { Female: } \mathrm{MD} \\
\text { Controls } \mathrm{n}= \\
30 \\
\text { Age: } 38.7 \pm \\
8.3 \text { years } \\
\text { Female: } \mathrm{MD}\end{array}$ & $\begin{array}{l}100 \% \text { HCQ } \\
3.3 \% \text { AZA } \\
3.3 \% \text { MTX }\end{array}$ & $\begin{array}{l}\nearrow \mathrm{PWV} \text { in SS } \\
\text { patients }(7.6 \pm 2.5 \\
\text { vs } 6.5 \pm 2.5, p= \\
0.003) .\end{array}$ \\
\hline
\end{tabular}

AIx: Augmentation Index; AZA: Azathioprine; CTC: Corticoids; HCQ: Hydroxychloroquine; (c)IMT: (carotid) Intima-Media Thickness; IS: Immunosuppressive drug; IQR: Interquartile Range; MD: Missing Data; MTX: Methotrexate; NSAID: Nonsteroidal Anti-Inflammatory Drug; OA: Osteoarthritis; PWV: Pulse Wave Velocity (hf: heart-femoral, cf.: carotid-femoral, hc: heart-carotid, br: brachialradial, fa: femoral-ankle); SS: Sjögren's Syndrome; SSDI: Sjögren's Syndrome Damage Index.

the Framingham Risk Score showed no difference between AnS patients and controls [74]. This illustrates that, again, traditional CV risk factors may not fully explain the increase in CVD in AnS, and, as with other inflammatory diseases, early detection of vascular dysfunction appears to be crucial.

Out of a total of 35 articles, involving 1535 patients with AnS and 1347 controls, a meta- analysis reported a significant increase in PWV in patients with AnS compared to controls [75]. In this meta-analysis, AIx was not documented. However, the results of several studies showed an increase in AIx in AnS patients compared with controls [76] [74]. Two studies found no association between arterial stiffness and AnS [10,77] but suffer from numerous biases such as a small sample size, a young population, a short duration of the disease and a high percentage of patients taking Non-Steroidal Anti-Inflammatory Drugs (NSAIDs) that may induce a low disease activity [77].

\subsubsection{Influence of disease characteristics and CV risk factors on arterial stiffness}

Disease activity appears to be associated with arterial stiffness. In a study of 85 AnS patients, AIx increased progressively with increasing CRP, erythrocyte sedimentation rate (ESR) and Ankylosing Spondylitis Disease Activity Score (ASDAS) [78]. In this study, baseline Bath Ankylosing Spondylitis Disease Activity Index (BASDAI) has not been associated with increased arterial stiffness, suggesting that objective assessment of disease activity such as ASDAS appears to be more relevant than disease activity measured only by BASDAI to evaluate CV risk. Finally, in a smaller cohort of 24 AnS patients, PWV and AIx were significantly associated with Bath Ankylosing Spondylitis Functional Index (BASFI) score [76].

\subsubsection{Influence of treatment on arterial stiffness}

A few studies focused on the effect of AnS treatment on arterial stiffness. When echocardiographic parameters were evaluated according to the different drug treatment groups, in $\mathbf{5 5}$ AnS patient, the aortic stiffness index was highest in those taking conventional synthetic Disease Modifying Anti-Rheumatic Drugs (csDMARDs) and was significantly higher in this group than in the control group and the TNFi group [79]. However, these results should be treated with caution. Indeed, AnS patients taking DMARDS had higher ESR and CRP levels, which may be a potential bias. Moreover, physical activity is a cornerstone in AnS treatment, and reduced cardiorespiratory fitness may induce an increased risk of CVD in AnS. Among 118 participating patients, a significant inverse association between $\mathrm{VO} 2$ peak and arterial stiffness was reported, independently of traditional $\mathrm{CV}$ risk factors and disease activity measures [80].

\subsubsection{Influence of arterial stiffness on cardiac function}

Myocardial involvement in AnS is usually manifested by diastolic dysfunction [81]. In a study of 75 patients with a mean disease duration of 5 years, AnS was associated with reduced LV strain in all myocardial layers [82].

\section{Conclusion}

Arterial stiffness is increased in chronic inflammatory and autoimmune diseases. This association is not solely related to the presence of the presence of $\mathrm{CV}$ risk factors. Therefore, CV risk assessment in these patients should not be based solely on the traditional CV risk scores but may also consider PWV and AIx.

However, CV risk factors, especially arterial hypertension, play a major role in arterial stiffness and should be controlled as early and as strictly as possible. Arterial stiffness is also clearly implicated in LV diastolic dysfunction, which is known to be the main mechanism of heart failure in chronic inflammatory condition. The link between arterial stiffness and atherosclerosis remains unclear in systemic inflammatory diseases. The lack of data and the high prevalence of CV risk factors make interpretation of the results difficult.

It remains unclear which characteristics of the disease may influence the presence or severity of the arterial aging and further studies or metaanalyses need to be conducted. To this end, indices which consider the burden of inflammatory disease rather than a measure of activity at a specific point in time would be useful.

TNFi appear to reduce arterial stiffness. One could therefore imagine that in the future, prescribing TNFi would be based not only on disease control but also on CV risk level. 
To summarize, this review highlights the relationship between arterial stiffness and chronic inflammatory and autoimmune diseases, supporting that CV risk assessment in inflammatory patients should also rely on PWV and AIx.

\section{Author contributions}

Conceptualization: Berger, Fesler, Roubille.

Data curation: Berger.

Formal analysis: Berger, Fesler, Roubille.

Funding acquisition: NA.

Investigation: Berger.

Methodology: Berger, Fesler, Roubille.

Project administration: Fesler, Roubille.

Resources; Software; Supervision; Validation: Fesler, Roubille.

Writing - original draft: Berger, Roubille.

Writing - review \& editing: Berger, Fesler, Roubille.

\section{Funding}

None.

\section{Declaration of Competing Interest}

MB has no conflict of interest to declare.

$\mathrm{PF}$ has received fees from Servier, and Actelion in the last 5 years. CR has received fees from Servier, and Pfizer in the last 5 years.

\section{References}

[1] Escárcega RO, Lipinski MJ, García-Carrasco M, Mendoza-Pinto C, GalvezRomero JL, Cervera R. Inflammation and atherosclerosis: cardiovascular evaluation in patients with autoimmune diseases. Autoimmun Rev 2018;17(7): 703-8.

[2] Crowson CS, Rollefstad S, Ikdahl E, Kitas GD, van Riel PLCM, Gabriel SE, et al. Impact of risk factors associated with cardiovascular outcomes in patients with rheumatoid arthritis. Ann Rheum Dis 2018;77(1):48-54.

[3] Jadhav UM, Kadam NN. Non-invasive assessment of arterial stiffness by pulsewave velocity correlates with endothelial dysfunction. Indian Heart J 2005;57(3): 226-32.

[4] Vlachopoulos C, Aznaouridis K, Stefanadis C. Prediction of cardiovascular events and all-cause mortality with arterial stiffness. J Am Coll Cardiol 2010;55(13): $1318-27$

[5] Laurent S, Cockcroft J, Van Bortel L, Boutouyrie P, Giannattasio C, Hayoz D, et al. Expert consensus document on arterial stiffness: methodological issues and clinical applications. Eur Heart J 2006;27(21):2588-605.

[6] Mackenzie IS, Wilkinson IB, Cockcroft JR. Assessment of arterial stiffness in clinical practice. QJM Mon J Assoc Physicians 2002;95(2):67-74.

[7] Jannasz I, Sondej T, Targowski T, Dobrowolski A, Olszewski R. Pulse wave velocity - a useful tool in assessing the stiffness of the arteries. Pol Merkur Lek Organ Pol Tow Lek 28 Juin 2019;46(276):257-62.

[8] Shirwany NA, Zou M. Arterial stiffness: a brief review. Acta Pharmacol Sin 2010; 31(10):1267-76.

[9] Yasmin. Similarities and differences between augmentation index and pulse wave velocity in the assessment of arterial stiffness. QJM 1 Oct 1999;92(10):595-600.

[10] Dzieża-Grudnik A, Sulicka J, Strach M, Siga O, Klimek E, Korkosz M, et al. Arteria stiffness is not increased in patients with short duration rheumatoid arthritis and ankylosing spondylitis. Blood Press 4 Mar 2017;26(2):115-21.

[11] Arida A, Zampeli E, Konstantonis G, Fragiadaki K, Kitas GD, Protogerou AD, et al Rheumatoid arthritis is sufficient to cause atheromatosis but not arterial stiffness or hypertrophy in the absence of classical cardiovascular risk factors. Clin Rheumatol 2015;34(5):853-9.

[12] Anyfanti P, Triantafyllou A, Gkaliagkousi E, Koletsos N, Aslanidis S, Douma S. Association of non-invasive hemodynamics with arterial stiffness in rheumatoid arthritis. Scand Cardiovasc J 4 juill 2018;52(4):171-6.

[13] Maloberti A, Riva M, Tadic M, Valena C, Villa P, Boggioni I, et al. Association between atrial, ventricular and vascular morphofunctional alterations in rheumatoid arthritis high blood press. Cardiovasc Prev 2018;25(1):97-104.

[14] Yang Y, Wang Z, Fu Z, Yang R, Wang J, Yuan L, et al. Stiffening of aorta is more preferentially associated with rheumatoid arthritis than peripheral arteries. Rheumatol Int 2019;39(10):1711-21.

[15] Vázquez-Del Mercado M, Gomez-Bañuelos E, Chavarria-Avila E, CardonaMuñoz E, Ramos-Becerra C, Alanis-Sanchez A, et al. Disease duration of rheumatoid arthritis is a predictor of vascular stiffness: a cross-sectional study in patients without known cardiovascular comorbidities. Medicine (Baltimore) août 2017;96(33):e7862.
[16] Özcan ANŞ, Aslan AN, Ünal Ö, Ercan K, Küçüksşahin O. A novel ultrasound-based technique to establish a correlation between disease activity and local carotid stiffness parameters in rheumatoid arthritis. Med Ultrason 10 Mai 2017;19(3): 288-94.

[17] Scanlon EM, Mankad R, Crowson CS, Kullo IJ, Mulvagh SL, Matteson EL, et al. Cardiovascular risk assessment in patients with rheumatoid arthritis: a correlative study of noninvasive arterial health testing. Clin Rheumatol 2017;36(4):763-71.

[18] Dregan A. Arterial stiffness association with chronic inflammatory disorders in the UK biobank study. Heart Br Card Soc 2018;104(15):1257-62.

[19] Aslan AN, Sirin Özcan AN, Erten S, Alsancak Y, Durmaz T. Assessment of local carotid stiffness in seronegative and seropositive rheumatoid arthritis. Scand Cardiovasc J 3 Sept 2017;51(5):255-60.

[20] Cioffi G, Viapiana O, Ognibeni F, Dalbeni A, Orsolini G, Adami S, et al. Clinical profile and outcome of patients with rheumatoid arthritis and abnormally high aortic stiffness. Eur J Prev Cardiol 2016;23(17):1848-59.

[21] Pieringer H, Pohanka E, Puchner R, Brummaier T. Association of vascular function and estimated cardiovascular risk in patients with rheumatoid arthritis. Rev Bras Reumatol 2017;57(5):452-60.

[22] Čypienè A, Dadonienè J, Miltinienẻ D, Rinkūnienè E, Rugienè R, Stropuvienè $S$, et al. The fact not to ignore: mean blood pressure is the main predictor of increased arterial stiffness in patients with systemic rheumatic diseases. Adv Med Sci 2017;62(2):223-9.

[23] Mitrović J, Morović-Vergles J, Horvatić I, Badžak J, Stojić M, Gamulin S, Ambulatory arterial stiffness index and carotid intima-media thickness in hypertensive rheumatoid patients: a comparative cross-sectional study. Int J Rheum Dis 2017;20(12):1998-2002.

[24] Li P, Han C, Ma C, Guo J, Liu B, Du J, et al. Determinants of brachial-ankle pulse wave velocity in Chinese patients with rheumatoid arthritis. Clin Dev Immunol 2013;2013:1-6.

[25] Mäki-Petäjä KM, Hall FC, Booth AD, Wallace SML, Null Yasmin, PWP Bearcroft, et al. Rheumatoid arthritis is associated with increased aortic pulse-wave velocity, which is reduced by anti-tumor necrosis factor-alpha therapy. Circulation 12 Sept 2006;114(11):1185-92.

[26] Mäki-Petäjä Kaisa M, Maysoon Elkhawad, Joseph Cheriyan, Joshi Francis R, Östör Andrew JK, Hall Frances C, et al. Anti-tumor necrosis factor- $\alpha$ therapy reduces aortic inflammation and stiffness in patients with rheumatoid arthritis. Circulation 20 Nov 2012;126(21):2473-80.

[27] Tam L-S, Shang Q, Li EK, Wang S, Li R-J, Lee K-L, et al. Infliximab is associated with improvement in arterial stiffness in patients with early rheumatoid arthritis — a randomized trial. J Rheumatol 1 Déc 2012;39(12):2267-75.

[28] Angel K, Provan SA, Fagerhol MK, Mowinckel P, Kvien TK, Atar D. Effect of 1year anti-TNF- $\alpha$ therapy on aortic stiffness, carotid atherosclerosis, and calprotectin in inflammatory arthropathies: a controlled study. Am J Hypertens 2012;25(6):644-50.

[29] Vassilopoulos D, Gravos A, Vlachopoulos C, Kandili A, Ioakeimidis N, Pectasides D, et al. Adalimumab decreases aortic stiffness independently of its effect in disease activity in patients with rheumatoid arthritis. Clin Rheumatol 2015;34(2):359-64.

[30] Vlachopoulos C, Gravos A, Georgiopoulos G, Terentes-Printzios D, Ioakeimidis N Vassilopoulos D, et al. The effect of TNF-a antagonists on aortic stiffness and wave reflections: a meta- analysis. Clin Rheumatol 2018;37(2):515-26.

[31] Novikova DS, Popkova TV, Lukina GV, Luchikhina EL, Karateev DE, Volkov AV, et al. The effects of rituximab on lipids, arterial stiffness and carotid intima-media thickness in rheumatoid arthritis. J Korean Med Sci 2016;31(2):202-7.

[32] Kume K, Amano K, Yamada S, Hatta K, Ohta H, Kuwaba N. Tocilizumab monotherapy reduces arterial stiffness as effectively as Etanercept or Adalimumab Monotherapy in rheumatoid arthritis: an open-label randomized controlled trial. J Rheumatol 2011;38(10):2169-71.

[33] Provan SA, Berg IJ, Hammer HB, Mathiessen A, Kvien TK, Semb AG. The impact of newer biological disease modifying anti-rheumatic drugs on cardiovascular risk factors: a 12- month longitudinal study in rheumatoid arthritis patients treated with rituximab, abatacept and tociliziumab. PLoS ONE 2015 Jun 26;10 (6):e0130709.

[34] Ursini F, Ruscitti P, Caio GPI, Manfredini R, Giacomelli R, De Giorgio R. The effect of non-TNF- targeted biologics on vascular dysfunction in theumatoid arthritis: a systematic literature review. Autoimmun Rev 2019;18(5):501-9.

[35] Wright K, Crowson CS, Gabriel SE. Cardiovascular comorbidity in rheumatic diseases: a focus on heart failure. Heart Fail Clin 2014;10(2):339-52.

[36] Ntusi NAB, Francis JM, Gumedze F, Karvounis H, Matthews PM, Wordsworth PB, et al. Cardiovascular magnetic resonance characterization of myocardial and vascular function in rheumatoid arthritis patients. Hell J Cardiol HJC Hell Kardiologike Epitheorese 2019;60(1):28-35.

[37] Gunter S, Robinson C, Woodiwiss AJ, Norton GR, Solomon A, Tsang L, et al. Arterial wave reflection and subclinical atherosclerosis in rheumatoid arthritis. Clin Exp Rheumatol 2018;9.

[38] Van Sij1 AM, Van Den Hurk K, Peters MJL, Van Halm VP, Nijpels G, Stehouwer CDA, et al. Different type of carotid Arterial Wall remodeling in rheumatoid arthritis compared with healthy subjects: a case-control study. J Rheumatol 2012;39(12):2261-6.

[39] Robustillo-Villarino M, Alegre-Sancho JJ, Rodilla-Sala E, Corrales A, Llorca J, Gonzalez-Gay MA, et al. Pulse wave velocity and augmentation index are not independently associated with carotid atherosclerosis in patients with rheumatoid arthritis. Clin Rheumatol 2017;36(11):2601-6.

[40] Wu G-C, Liu H-R, Leng R-X, Li X-P, Li X-M, Pan H-F, et al. Subclinical atherosclerosis in patients with systemic lupus erythematosus: a systemic review and meta-analysis. Autoimmun Rev 2016;15(1):22-37. 
[41] Chazal T, Kerneis M, Guedeney P, Haroche J, Mathian A, Rufat P, et al. Coronary artery disease in systemic lupus: a case-controlled angiographic study. Autoimmun Rev Janv 2020;19(1):102427.

[42] Montalbán-Méndez C, Soriano-Maldonado A, Vargas-Hitos JA, Sáez-Urán LM, Rosales-Castillo A, Morillas-de-Laguno P, et al. Cardiorespiratory fitness and agerelated arterial stiffness in women with systemic lupus erythematosus. Eur J Clin Invest 2018;48(3):e12885.

[43] Kirchler C, Husar-Memmer E, Rappersberger K, Thaler K, Fritsch-Stork R. Type I Interferon as cardiovascular risk factor in systemic and cutaneous lupus erythematosus: a systematic review. Autoimmun Rev 17 Mars 2021:102794.

[44] Iuliano A, Galeazzi M, Sebastiani GD. Antiphospholipid syndrome's genetic and epigenetic aspects. Autoimmun Rev Sept 2019;18(9):102352.

[45] Valero-Gonzalez S, Castejon R, Jimenez-Ortiz C, Rosado S, Tutor-Ureta P, Vargas J-A, et al. Increased arterial stiffness is independently associated with metabolic syndrome and damage index in systemic lupus erythematosus patients. Scand J Rheumatol 2014;43(1):54-8.

[46] Tziomalos K, Gkougkourelas I, Sarantopoulos A, Bekiari E, Makri E, Raptis N, et al. Arterial stiffness and peripheral arterial disease in patients with systemic lupus erythematosus. Rheumatol Int 2017;37(2):293-8.

[47] Stortz M, Triantafyllias K, Schwarting A, Weinmann-Menke J. Vascular stiffness: influencing factors on carotid-femoral pulse wave velocity in systemic lupus erythematosus. Clin Exp Rheumatol 2020;38(1):74-81.

[48] Wang P, Mao Y-M, Zhao C-N, Liu L-N, Li X-M, Li X-P, et al. Increased pulse wave velocity in systemic lupus Erythematosus: a Meta-analysis. Angiology 2018;69(3): 228-35.

[49] Ding FM, Li M, Yang X, Ye Y, Kang L, Pang H, et al. Accelerated age-related arterial stiffness in systemic lupus Erythematosus patients. J Clin Rheumatol Pract Rep Rheum Musculoskelet Dis 2016;22(8):426-33.

[50] Parra S, Lopez-Dupla M, Ibarretxe D, de Las Heras M, Amigó N, Català A, et al. Patients with systemic lupus Erythematosus show an increased arterial stiffness that is predicted by IgM anti- 32 -glycoprotein I and small dense high-density lipoprotein particles. Arthritis Care Res 2019;71(1):116-25.

[51] Karp G, Wolak A, Baumfeld Y, Bar-Am N, Novack V, Wolak T, et al. Assessment of aortic stiffness among patients with systemic lupus erythematosus and rheumatoid arthritis by magnetic resonance imaging. Int J Cardiovasc Imaging 2016;32(6):935-44.

[52] Sacre K, Escoubet B, Pasquet B, Chauveheid M-P, Zennaro M-C, Tubach F, et al. Increased arterial stiffness in systemic lupus erythematosus (SLE) patients at low risk for cardiovascular disease: a cross-sectional controlled study. PLoS One 2014; 9(4):e94511.

[53] Santos MJ, Carmona-Fernandes D, Canhāo H, Canas da Silva J, Fonseca JE, Gil V. Early vascular alterations in SLE and RA patients-a step towards understanding the associated cardiovascular risk. PLoS One 2012;7(9):e44668.

[54] Mercurio V, Lobasso A, Barbieri L, Parrella P, Ciervo D, Liccardo B, et al. Inflammatory, serological and vascular determinants of cardiovascular disease in systemic lupus erythematosus patients. Int J Mol Sci 30 Avr 2019;20(9),

[55] Jurcut C, Caraiola S, Nitescu D, Jurcut R, Giusca S, Baicus C, et al. Subclinical vascular disease in patients with systemic lupus erythematosus: the additive deleterious effect of the antiphospholipid syndrome. Jt Bone Spine Rev Rhum 2012;79(6):628-9.

[56] Sezis Demirci M, Karabulut G, Gungor O, Celtik A, Ok E, Kabasakal Y. Is there an increased arterial stiffness in patients with primary Sj\&ouml;gren's syndrome? Intern Med 2016;55(5):455-9.

[57] Sabio JM, Sánchez-Berná I, Martinez-Bordonado J, Vargas-Hitos JA, NavarreteNavarrete N, Ruíz ME, et al. Prevalence of and factors associated with increased arterial stiffness in patients with primary Sjögren's syndrome. Arthritis Care Res 2015;67(4):554-62.

[58] Gyöngyösi M, Pokorny G, Jambrik Z, Kovács L, Kovács A, Makula E, et al. Cardiac manifestations in primary Sjögren's syndrome. Ann Rheum Dis 1 Juill 1996;55 (7):450-4.

[59] Atzeni F, Sarzi-Puttini P, Signorello MC, Gianturco L, Stella D, Boccassini L, et al. New parameters for identifying subclinical atherosclerosis in patients with primary Sjögren's syndrome: a pilot study. Clin Exp Rheumatol May-Jun 2014;32 (3):361-8

[60] Haroon M, Gallagher P, Heffernan E, FitzGerald O. High prevalence of metabolic syndrome and of insulin resistance in psoriatic arthritis is associated with the severity of underlying disease. J Rheumatol 2014;41(7):1357-65.

[61] Langan SM, Seminara NM, Shin DB, Troxel AB, Kimmel SE, Mehta NN, et al Prevalence of metabolic syndrome in patients with psoriasis: a population-based study in the United Kingdom. J Invest Dermatol 2012;132(3 Pt 1):556-62.

[62] Costa L, Caso F, D'Elia L, Atteno M, Peluso R, Del Puente A, et al. Psoriatic arthritis is associated with increased arterial stiffness in the absence of known cardiovascular risk factors: a case control study. Clin Rheumatol 2012;31(4): $711-5$.

[63] Patschan D, Sugiarto N, Henze E, Mößner R, Mohr J, Müller GA, et al. Early endothelial progenitor cells and vascular stiffness in psoriasis and psoriatic arthritis. Eur J Med Res 9 Nov 2018;23(1):56.

[64] Balta I, Balta S, Demirkol S, Celik T, Ekiz O, Cakar M, et al. Aortic arterial stiffness is a moderate predictor of cardiovascular disease in patients with psoriasis vulgaris. Angiology 2014;65(1):74-8.

[65] Sunbul M, Seckin D, Durmus E, Ozgen Z, Bozbay M, Bozbay A, et al. Assessment of arterial stiffness and cardiovascular hemodynamics by oscillometric method in psoriasis patients with normal cardiac functions. Heart Vessels 2015;30(3): 347-54.

[66] Altekin ER, Koç S, Karakaș MS, Yanıkoğlı A, Başarıcı I, Demir I, et al Determination of subclinical atherosclerosis in plaque type psoriasis patients without traditional risk factors for atherosclerosis. Turk Kardiyol Dernegi Arsivi Turk Kardiyol Derneginin Yayin Organidir 2012;40(7):574-80.

[67] Dattilo G, Imbalzano E, Casale M, Guarneri C, Borgia F, Mondello S, et al. Psoriasis and cardiovascular risk: correlation between psoriasis and cardiovascular functional indices. Angiology 2018;69(1):31-7.

[68] Ikonomidis I, Makavos G, Papadavid E, Varoudi M, Andreadou I, Gravanis K, et al. Similarities in coronary function and myocardial deformation between psoriasis and coronary artery disease: the role of oxidative stress and inflammation. Can J Cardiol 2015;31(3):287-95.

[69] de Oliveira AN, Simões MM, Simões R, MVB Malachias, Rezende BA. Cardiovascular risk in psoriasis patients: clinical, functional and morphological parameters. Arq Bras Cardiol 2019;113(2):242-9.

[70] Hansen PR, Isaksen JL, Jemec GB, Ellervik C, Kanters JK. Arterial stiffness in subjects with psoriasis: a cross-sectional population study. Eur J Dermatol EJD 1 Oct 2018;28(5):683-5.

[71] Shen J, Shang Q, Li EK, Leung Y-Y, Kun EW, Kwok L-W, et al. Cumulative inflammatory burden is independently associated with increased arterial stiffness in patients with psoriatic arthritis: a prospective study. Arthritis Res Ther 17 Mars 2015;17:75.

[72] Shang O, Tam L-S, Sanderson JE, Sun J-P, Li EK-M, Yu C-M. Increase in ventricular-arterial stiffness in patients with psoriatic arthritis. Rheumatology 1 Déc 2012;51(12):2215-23.

[73] Castañeda S, Nurmohamed MT, González-Gay MA. Cardiovascular disease in inflammatory rheumatic diseases. Best Pract Res Clin Rheumatol 2016;30(5): 851-69.

[74] Berg IJ, van der Heijde D, Dagfinrud H, Seljeflot I, Olsen IC, Kvien TK, et al. Disease activity in ankylosing spondylitis and associations to markers of vascular pathology and traditional cardiovascular disease risk factors: a cross-sectional study. J Rheumatol 2015;42(4):645-53.

[75] Bai R, Zhang Y, Liu W, Ma C, Chen X, Yang J, et al. The relationship of Ankylosing spondylitis and subclinical atherosclerosis: a systemic review and meta-analysis. Angiology 2019;70(6):492-500.

[76] Avram C, Drăgoi RG, Popoviciu H, Drăgoi M, Avram A, Amaricăi E. Association between arterial stiffness, disease activity and functional impairment in ankylosing spondylitis patients: a cross- sectional study. Clin Rheumatol 2016;35 (8):2017-22.

[77] Kaplanoglu H, Özișler C. Evaluation of subclinical atherosclerosis using ultrasound radiofrequency data technology in patients diagnosed with ankylosing spondylitis. J Ultrasound Med Off J Am Inst Ultrasound Med 2019;38(3):703-11.

[78] Berg IJ, Semb AG, van der Heijde D, Kvien TK, Olsen IC, Dagfinrud H, et al. CRP and ASDAS are associated with future elevated arterial stiffness, a risk marker o cardiovascular disease, in patients with ankylosing spondylitis: results after 5year follow-up. Ann Rheum Dis 2015;74(8):1562-6.

[79] Ozen S, Ozen A, Unal EU, Tufekcioglu O, Ataman S, Yalcin AP. Subclinical cardiac disease in ankylosing spondylitis. Echocardiogr Mt Kisco N 2018:35(10): $1579-86$.

[80] Berg IJ, Semb AG, Sveaas SH, Fongen C, van der Heijde D, Kvien TK, et al. Associations between cardiorespiratory fitness and arterial stiffness in Ankylosing spondylitis: a cross-sectional study. J Rheumatol 2018;45(11):1522-5.

[81] Okan T, Sari I, Akar S, Cece H, Goldeli O, Guneri S, et al. Ventricular diastolic function of ankylosing spondylitis patients by using conventional pulsed wave Doppler, myocardial performance index and tissue Doppler imaging. Echocardiogr Mt Kisco N 2008;25(1):47-56.

[82] Ozkaramanli Gur D, Ozaltun DN, Guzel S, Sarifakioglu B, Akyuz A, Alpsoy S, et al Novel imaging modalities in detection of cardiovascular involvement in ankylosing spondylitis. Scand Cardiovasc J SCJ 2018;52(6):320-7.

[83] Gunter S, Robinson C, Norton GR, Woodiwiss AJ, Tsang L, Dessein PH, et al, Cardiovascular risk factors and disease characteristics are consistently associated with arterial function in rheumatoid arthritis. J Rheumatol 2017:44(8):1125-33.

[84] Ikdahl E, Rollefstad S, Wibetoe G, Olsen IC, Berg I-J, Hisdal J, et al. Predictive value of arterial stiffness and subclinical carotid atherosclerosis for cardiovascular disease in patients with rheumatoid arthritis. J Rheumatol 2016; 43(9):1622-30.

[85] Karakulak UN, Sahiner L, Maharjan N, Okutucu S, Evranos B, Aladag E, et al. Evaluation of the ambulatory arterial stiffness index in patients with rheumatoid arthritis. Blood Press Monit 2015;20(5):254-9.

[86] Pieringer H, Brummaier T, Schmid M, Pichler M, Hayat-Khayyati A, Ebner S, et al Rheumatoid arthritis is an independent risk factor for an increased augmentation index regardless of the coexistence of traditional cardiovascular risk factors. Semin Arthritis Rheum 2012;42(1):17-22.

[87] Tam L-S, Shang Q, Li EK, Wong S, Li R-J, Lee K-L, et al. Serum soluble receptor for advanced glycation end products levels and aortic augmentation index in early rheumatoid arthritis-a prospective study. Semin Arthritis Rheum 2013;42(4): 333-45.

[88] Kim Y-S, Sung Y-K, Choi C-B, Uhm W-S, Kim T-H, Shin J-H, et al. The major determinants of arterial stiffness in Korean patients with rheumatoid arthritis are age and systolic blood pressure, not disease-related factors. Rheumatol Int 2012; 32(11):3455-61.

[89] Ilter A, Kiris A, Karkucak M, Sahin M, Serdar OF, Ugan Y. Arterial stiffness is associated with left ventricular dysfunction in patients with rheumatoid arthritis. Clin Rheumatol 2016;35(11):2663-8.

[90] Byram KW, Oeser AM, Linton MF, Fazio S, Stein CM, Ormseth MJ. Exercise is associated with increased small HDL particle concentration and decreased vascular stiffness in rheumatoid arthritis. J Clin Rheumatol Pract Rep Rheum Musculoskelet Dis 2018:24(8):417-21. 
[91] Woodman RJ, Baghdadi LR, Shanahan EM, de Silva I, Hodgson JM, Mangoni AA. Diets high in $\mathrm{n}-3$ fatty acids are associated with lower arterial stiffness in patients with rheumatoid arthritis: a latent profile analysis. Br J Nutr 2019;121(2): 182-94.

[92] Mathieu S, Pereira B, Dubost J-J, Lusson J-R, Soubrier M. No significant change in arterial stiffness in RA after 6 months and 1 year of rituximab treatment. Rheumatol Oxf Eng1 2012;51(6):1107-11.

[93] Gkaliagkousi E, Anyfanti P, Chatzimichailidou S, Triantafyllou A, Lazaridis A Aslanidis S, et al. Association of nocturnal blood pressure patterns with inflammation and central and peripheral estimates of vascular health in rheumatoid arthritis. J Hum Hypertens 2018;32(4):259-67.

[94] Botta E, Meroño T, Saucedo C, Martín M, Tetzlaff W, Sorroche P, et al. Associations between disease activity, markers of HDL functionality and arterial stiffness in patients with rheumatoid arthritis. Atherosclerosis 2016;251:438-44.

[95] Daïen CI, Fesler P, du Cailar G, Daïen V, Mura T, Dupuy A-M, et al. Etanercept normalises left ventricular mass in patients with rheumatoid arthritis. Ann Rheum Dis 2013;72(6):881-7.

[96] Angel K, Provan SA, Hammer HB, Mowinckel P, Kvien TK, Atar D. Changes in arterial stiffness during continued infliximab treatment in patients with inflammatory arthropathies: long-term anti-TNF- $\alpha$ therapy and arterial stiffness. Fundam Clin Pharmacol 2011;25(4):511-7.

[97] Morreale M, Mulè G, Ferrante A, D'ignoto F, Cottone S. Early vascular aging in normotensive patients with systemic lupus Erythematosus: comparison with young patients having hypertension. Angiology. 2016;67(7):676-82.

[98] Kim SY, Yang HS, Lee YW, Choe YB, Ahn KJ. Evaluation of the Beta stiffness index and carotid intima-media thickness in Asian patients with psoriasis. Angiology 2015;66(9):889-95.

[99] Di Vito L, Abeni D, Uras C, Magenta A, Ciarapica R, Capogrossi MC, et al. Role of psoriasis on subclinical cardiovascular disease. Minerva Med 2018;109(3):255-8

[100] Biesbroek PS, Heslinga SC, van de Ven PM, Peters MJL, Amier RP, Konings TC, et al. Assessment of aortic stiffness in patients with ankylosing spondylitis using cardiovascular magnetic resonance. Clin Rheumatol 2018;37(8):2151-9.

[101] Zardi EM, Sambataro G, Basta F, Margiotta DPE, Afeltra AMV. Subclinical carotid atherosclerosis in elderly patients with primary Sjögren syndrome: a duplex Doppler Sonographic study. Int J Immunopathol Pharmacol 2014;27(4):645-51.

[102] Ozisler C, Kaplanoglu H. Evaluation of subclinical atherosclerosis by ultrasound radiofrequency data technology in patients with primary Sjögren's syndrome. Clin Rheumatol 2019;38(3):709-17. 